\title{
LHAPDF6: parton density access in the LHC precision era
}

\author{
Andy Buckley ${ }^{1, a}$, James Ferrando ${ }^{1}$, Stephen Lloyd ${ }^{2}$, Karl Nordström ${ }^{1}$, Ben Page ${ }^{3}$, Martin Rüfenacht ${ }^{4}$, \\ Marek Schönherr ${ }^{5}$, Graeme Watt ${ }^{6}$ \\ ${ }^{1}$ School of Physics and Astronomy, University of Glasgow, Glasgow, UK \\ ${ }^{2}$ School of Physics and Astronomy, University of Edinburgh, Edinburgh, UK \\ ${ }^{3}$ Departamento de Física Teórica y del Cosmos y CAFPE, Universidad de Granada, Granada, Spain \\ ${ }^{4}$ School of Informatics, University of Edinburgh, Edinburgh, UK \\ ${ }^{5}$ Physik-Institut, Universität Zürich, Zurich, Switzerland \\ ${ }^{6}$ Institute for Particle Physics Phenomenology, Durham University, Durham, UK
}

Received: 26 December 2014 / Accepted: 10 February 2015 / Published online: 20 March 2015

(C) The Author(s) 2015. This article is published with open access at Springerlink.com

\begin{abstract}
The Fortran LHAPDF library has been a longterm workhorse in particle physics, providing standardised access to parton density functions for experimental and phenomenological purposes alike, following on from the venerable PDFLIB package. During Run 1 of the LHC, however, several fundamental limitations in LHAPDF's design have became deeply problematic, restricting the usability of the library for important physics-study procedures and providing dangerous avenues by which to silently obtain incorrect results. In this paper we present the LHAPDF6 library, a ground-up re-engineering of the PDFLIB/LHAPDF paradigm for PDF access which removes all limits on use of concurrent PDF sets, massively reduces static memory requirements, offers improved CPU performance, and fixes fundamental bugs in multi-set access to PDF metadata. The new design, restricted for now to interpolated PDFs, uses centralised numerical routines and a powerful cascading metadata system to decouple software releases from provision of new PDF data and allow completely general parton content. More than 200 PDF sets have been migrated from LHAPDF 5 to the new universal data format, via a stringent quality control procedure. LHAPDF 6 is supported by many Monte Carlo generators and other physics programs, in some cases via a full set of compatibility routines, and is recommended for the demanding PDF access needs of LHC Run 2 and beyond.
\end{abstract}

\section{Contents}

1 Introduction . . . . . . . . . . . . . . 2

1.1 Definitions and conventions . . . . . . . . 2

a e-mail: andy.buckley@cern.ch
2 History and evolution of LHAPDF . . . . . . . . 3

2.1 Performance problems . . . . . . . . . . 3

2.2 Correctness problems . . . . . . . . . . . 4

2.3 Maintainability problems . . . . . . . . 4

2.4 Summary of LHAPDF 5 issues . . . . . . . 4

3 Design of LHAPDF $6 \ldots \ldots \ldots$

3.1 PDF value access . . . . . . . . . . 4

3.2 PDF metadata . . . . . . . . . . . . . 5

3.3 Object and memory management . . . . . . 5

3.4 PDF value calculation . . . . . . . . . . . 5

3.4.1 Interpolator system . . . . . . . . 6

3.4.2 Extrapolation system . . . . . . . . 6

$3.5 \alpha_{\mathrm{S} \text { system } \ldots \ldots \ldots \ldots \ldots} \ldots \ldots \ldots \ldots$

4 Usage examples . . . . . . . . . . . . . . . . . . 8

$4.1 \quad \mathrm{C}++$ example . . . . . . . . . . . . . . 8

4.2 Python example . . . . . . . . . . . . 8

4.3 Fortran example (same as for LHAPDF 5) . . 8

5 Data formats .............. . . 8

5.1 Metadata format . . . . . . . . . . . . 8

5.1.1 System-level metadata . . . . . . . . . 8

5.1.2 Set-level metadata . . . . . . . . . . . 9

5.1.3 Member-level metadata . . . . . . . 10

5.2 PDF grid data format . . . . . . . . . . 10

$5.3 \alpha_{\mathrm{S}}$ interpolation data format . . . . . . . . . 10

5.4 Index file . . . . . . . . . . . . . . 11

5.5 Distribution and updating . . . . . . . . . 11

6 PDF uncertainties . . . . . . . . . . . . 11

6.1 set.uncertainty (values, cl, alternative) ........... 12

6.2 set.correlation(valuesA, valuesB) . 12

6.3 set.randomValueFromHessian(values, randoms, symmetrise)...... 13

7 PDF reweighting . . . . . . . . . . 13

8 LHAPDF 5/PDFLIB compatibility . . . . . . . . 14 
8.1 Legacy code interfaces . . . . . . . . . . . 14

8.2 Version detection hooks . . . . . . . . . . . 14

8.3 Uptake and prospects . . . . . . . . . . 15

9 Benchmarking and performance . . . . . . . 15

9.1 Memory requirements . . . . . . . . 15

9.2 CPU performance . . . . . . . . . . 15

9.3 Disk space requirements . . . . . . . . 17

10 PDF migration and validation . . . . . . . . . . 17

11 Summary and prospects . . . . . . . . . . . 17

References . . . . . . . . . . . . . . 19

\section{Introduction}

Parton density functions (PDFs) are a crucial input into crosssection calculations at hadron colliders; they encode the process-independent momentum structure of partons within hadrons, with which partonic cross-sections must be convolved to obtain physical results that can be compared to experimental data. At leading order in perturbation theory, PDFs encode the probability that a beam hadron's momentum is carried by a parton of given flavour and momentum fraction. At higher orders this interpretation breaks down and positivity is no longer required - but PDF normalization at all orders is constrained by the requirement that a sum over all parton flavours $i$ and momentum fractions $x$ equates to the whole momentum of the incoming beam hadron $B$ :

$\sum_{i} \int_{0}^{1} \mathrm{~d} x \times f_{i / B}\left(x ; Q^{2}\right)=1$,

where $f_{i / B}\left(x ; Q^{2}\right)$ is the parton density function for parton $i$ in $B$, at a factorization scale $Q$. Conservation of baryon number leads to a flavour sum rule,

$\int_{0}^{1} \mathrm{~d} x\left(f_{i / B}\left(x ; Q^{2}\right)-\bar{f}_{i / B}\left(x ; Q^{2}\right)\right)=n_{i}$,

where $i$ runs over quark flavours and $\bar{f}_{i / B}$ is the antiquark PDF in baryon $B$. For protons, $n_{u}=2, n_{d}=1$, and $n_{\{s, c, b, t\}}=0$.

Parton density calculations sit astride the borderline of perturbative and non-perturbative QCD, constructed by fitting of a factorised low-scale, non-perturbative component to experimental data and then evolved to higher scales using perturbative QCD running, most commonly DGLAP evolution. In general, PDFs may include a transverse momentum dependence but here we restrict ourselves to collinear PDFs where the extracted parton momenta are perfectly aligned with that of the parent hadron; such PDFs are then defined as a two-variable function $f_{i / B}(x ; Q)$ for collinear momentum fraction $x$ and factorization scale $Q$. Equations (1) and (2) apply independently at each value of $Q$, hence the semicolon separator between $f$ 's parameters.

The LHAPDF library is the ubiquitous means by which parton density functions are accessed for LHC experimental and phenomenological studies. It is both a framework for uniform access to the results of many different PDF fitting groups and a collection of such PDF sets. The first version of LHAPDF was developed to solve scaling problems with the previously standard PDFLIB library [1], and to retain backward compatibility with it; in this paper we describe a similar evolution within the LHAPDF package, from a Fortran-based static memory paradigm to a $\mathrm{C}++$ one in which dynamic $\mathrm{PDF}$ object creation, concurrent usage, and removal of artificial limitations are fundamental. This new version addresses the most serious limitations of the Fortran version, permitting a new level complexity of PDF systematics estimation for precision physics studies at the LHC [2] Run 2 and beyond.

\subsection{Definitions and conventions}

Since the beam hadron will in most current applications be a proton, we will simplify the notation from here by dropping the / $B$ specification of the parent hadron, i.e. $f_{i}\left(x ; Q^{2}\right)$ rather than $f_{i / B}\left(x ; Q^{2}\right)$. Other parent hadrons are possible, of course, notably neutrons which can either be fitted explicitly or obtained from proton PDFs assuming strong isospin symmetry.

The PDFs appear in hadron collider cross-section calculations in the form $[3,4]$ :

$\sigma=\int \mathrm{d} x_{1} \mathrm{~d} x_{2} f_{i}\left(x_{1} ; Q^{2}\right) f_{j}\left(x_{2} ; Q^{2}\right) \hat{\sigma}_{i j}\left(x_{1}, x_{2}, Q^{2}\right)$,

where $\hat{\sigma}_{i j}$ is the partonic cross-section for a process with incoming partons $i$ and $j$. Usually several partonic initial states contribute and should be summed over in Eq. (3).

Given the fundamental role played by the $x f\left(x ; Q^{2}\right)$ structure in the fitting and use of PDFs, it is this form which is encoded in the LHAPDF library. We will tend to refer to this encoded value as the "PDF value" or similar, even though it is in fact a combination of the parton density function and the momentum fraction $x$.

Another ambiguity in common usage is the meaning of the words "PDF set", which are sometimes used interchangeably with "PDF" and sometimes not. If one considers a PDF to be a function defined for a given parton flavour, then both a collection of such functions for all flavours, and a larger collection of systematic variations on such collections can reasonably be called a "PDF set". In this paper, particularly when referring to LHAPDF code objects, we will take the approach that a "PDF" or "PDF set member" is a complete set of 1-flavour parton density functions; we refer to a larger collection of 
systematic variations on such an object, e.g. eigenvectors or Monte Carlo (MC) replicas, as a "PDF set".

Finally, when referring to code objects or configuration directives we will do so in typewriter font.

\section{History and evolution of LHAPDF}

LHAPDF versions 5 and earlier [5,6] arose out of the 2001 Les Houches "Physics at TeV Colliders" workshop [7], as the need for a scalable system to replace PDFLIB became pressing. The main problem with PDFLIB was that the data for interpolating each PDF was stored in the library, and as PDF fitting became industrialised (particularly with the rise of the CTEQ and MRST error sets), this model was no longer viable.

LHAPDF was originally intended to address this problem by instead storing only the parameters of each parton density fit at a fixed low scale and then using standard DGLAP evolution in $Q$ via QCDNUM [8] to dynamically build an interpolation grid to higher scales, and thereafter work as before. However, by the mid-2000s and version 4 of LHAPDF, this model had also broken down. Each PDF parameterisation required custom code to be included in the LHAPDF library, and the bundled QCDNUM within LHAPDF had itself become significantly outdated: upgrading it was not an option due to the need for consistent behaviour between LHAPDF versions. PDF fitting groups, concerned that the built-in QCDNUM evolution would not precisely match that used by their own fitting code, universally chose to supply full interpolation grid files rather than evolution starting conditions, and as a result LHAPDF acquired a large collection of routines to read and use these data files in a myriad of formats.

At the same time as these trends back to interpolationbased PDF provision, user demand resulted in new features for simultaneous use of several PDF sets - the so-called "multiset" mode introduced in LHAPDF 5.0. The implementation of this was relatively trivial: the amount of allocated interpolation space was multiplied by a factor of NMXSET (with a default value of 3), but while it permitted rapid switching between a few concurrent sets the multiset mode did not integrate seamlessly with the original interface, potentially leading to incorrect results, and was memory-inefficient and limited in scalability.

\subsection{Performance problems}

The major problems with LHAPDF v5 relate to the technical implementation of the various interpolation routines and the multiset mode.

Both these issues are rooted in Fortran's static memory allocation. As usual, the interpolation routines for various PDFs operate on large arrays of floating point data. These were typically declared as Fortran common blocks, but in practice were not used commonly: each PDF group's "wrapper" code operates on its own array. As the collection of supported PDF sets became larger, the memory requirements of LHAPDF continually grew, and with version 5.9.1 (the final version in the v5 series) more than $2 \mathrm{~GB}$ was declared as necessary to use it at all. In practice operating systems did not allocate the majority of this uninitialised memory, but it proved a major issue for use of LHAPDF on the LHC Computing Grid system where static memory restrictions had to be passed in order for a job to run.

A workaround solution was provided for this problem: a so-called "low memory" build-time configuration which reduced the static memory footprint within acceptable limits, but at the heavy cost of only providing interpolation array space for one member in each PDF set. This mode is usually sufficient for event generation, in which only a single PDF is used, and in this form it was used for the LHC experimental collaborations' MC sample production through LHC Run 1. But it is incompatible with "advanced" PDF uncertainty studies in which each event must be re-evaluated or reweighted to every member in the PDF error set: constant re-initialisation of the single PDF slots from the data file slows operations to a crawl. For this reason, and because the low-memory mode is a build-time rather than run-time option, PDF reweighting studies for the LHC needed to use special, often private, user builds of LHAPDF with the attendant danger of inconsistency.

The era of the low-memory mode's suitability for event generation has also come to an end between LHC Runs 1 and 2, with the rise of next-to-leading order (NLO) matrix element calculations "matched" to parton shower algorithms $[9,10]$. The "NLO revolution" has been a great success of LHC-era phenomenology and the bulk of Standard Model processes are now simulated at fully-exclusive NLO - but the flip-side is that PDF reweightings now require detailed information about initial parton configurations in each NLO subtraction counter-term [11]. Accordingly PDF uncertainties are increasingly calculated as event weightings during the generation rather than retrospectively as done in the past for leading-order (LO) processes. ${ }^{1}$

Further options exist for selective disabling of LHAPDF support for particular PDF families, as an alternative way to reduce the memory footprint. However, since this highly restricts the parton density fits which can be used, it has not found much favour.

Of course, with a design so dependent on global state and shared memory, Fortran LHAPDF is entirely unsafe for

\footnotetext{
${ }_{1}^{1}$ NLO event generators may report summary PDF information, for example in HepMC's Pdf Info object, but this is an approximation and may give very misleading effects if used for retrospective reweighting.
} 
use in multi-threaded applications: this greatly restricts its scalability in the current multi-core computing era.

\subsection{Correctness problems}

The last set of problems with LHAPDF 5 relate, concerningly, to the correctness of the output. For example different generations of PDF fit families share the same interpolation code, although they may have different ranges of validity in $x-Q$ phase space, and wrong ranges are sometimes reported.

The reporting of $\Lambda_{\mathrm{QCD}}$ and other metadata has also been problematic, to the extent that PYTHIA6's many tunes depend on LHAPDF returning a nonsense value which is then reset to the default of $0.192 \mathrm{GeV}$. Since the multiset mode is often only implemented as a multiplying factor on the size and indexing offsets, reported values of metadata such as $\alpha_{\mathrm{S}}$ and $x \& Q$ boundaries do not always correspond to the currently active PDF slot, but rather to properties of the last set to have been initialised.

\subsection{Maintainability problems}

Aside from the technical issues discussed above, the design of LHAPDF 5 (and earlier versions) tightly couples PDF availability to the release cycle of the LHAPDF code library as in PDFLIB. As PDF fitting has become more diverse, with many different groups releasing PDF fits in response to new LHC and other data, the mismatch of the slow software releases (typically two releases per year) and the faster, less predictable release rate of new PDF sets has become evident. It is neither desirable for new PDF data to have to wait for months before becoming publicly available via an LHAPDF release, nor for experiments and other users to be deluged with new software versions to be installed and tested.

In addition, since adding new PDFs involved interfacing external Fortran code via "wrapper" routines, it both required significant coding and testing work from the LHAPDF maintainers, and blocked PDF fitting groups from using languages other than Fortran for their fitting/interpolation codes. The (partial) sharing of wrapper routines between some sets which did not provide their own interpolation code made any changes to existing wrapper code dangerous and fragile. An attempt was made to make it easier for users to make custom PDFs by using one of three generic set names to trigger a polynomial spline interpolation, but this was also very restricted in functionality and saw minimal use.

A final logistical issue was the lack of version tracking in PDF data files, which would periodically be found to be buggy, and no way to indicate which versions of the LHAPDF library were required to use a particular PDF. This led to some problems where for space-saving reasons PDF data would be shared between different versions of the library, producing unintended numerical changes and potentially introducing buggy outputs from previously functional installations.

\subsection{Summary of LHAPDF 5 issues}

Many of the problems of LHAPDF 5 stem from the combination of the static nature of Fortran memory handling and from the way that evolving user demands on LHAPDF forced retro-fitting of features such as grid interpolation and multiset mode on to a system not originally designed to incorporate them. These have combined with more logistical features such as the lack of any versioned connection between the PDF data files and the library, the menagerie of interpolation grid formats, and the need to modify the library to use a new PDF to make LHAPDF 5 difficult both to use and to maintain. These issues became critical during Run 1 of the LHC, leading to the development of LHAPDF 6 to deal with the increased demands on parton density usage in Run 2 and beyond. Version 5.9.1 of LHAPDF was the last in the Fortran series; all new development and maintenance (including provision of new PDF sets) is restricted to LHAPDF 6 only.

\section{Design of LHAPDF 6}

LHAPDF 6 is a ground-up redesign and re-implementation of the LHAPDF system, specifically to address all the above problems of the Fortran LHAPDF versions. As so many of these problems fundamentally stem from Fortran(77) static memory limitations, and the bulk of new experimental and event generator code is written in $\mathrm{C}++$, we have also chosen to write the new LHAPDF 6 in object oriented $\mathrm{C}++$. Since the Python scripting language has also become widely used in high-energy physics, we also provide a Python interface to the C++ LHAPDF library, which can be particularly useful for interactive PDF testing and exploration.

\subsection{PDF value access}

The central code/design object in LHAPDF 6 is the PDF, an interface class representing parton density functions for several parton flavours, typically but not necessarily the gluon plus the lightest 5 quark (and anti-quark) flavours. An extra object, PDFSet is provided purely for (significant) convenience in accessing PDF set metadata and all the members in the set, e.g. for making systematic variations within a set. The set level of data grouping is unavoidable, even in the case of single-member sets, and a list of all available PDF sets on the user's system can be obtained via the LHAPDF: : availablePDFSets () function. There is no LHAPDF 6 user-interface type to represent a single-flavour parton density.

Unlike in LHAPDF 5, where a few PDFs included a parton density for a non-standard flavour such as a photon or gluino 
via a special-case "hack" $[12,13]$, LHAPDF 6 allows completely general flavours, identified using the standard PDG Monte Carlo ID code [14] scheme. An alias of 0 for $21=$ gluon is also supported, for backward compatibility and the convenience of being able to access all QCD partons with a for-loop from -6 to 6 .

$x f\left(x ; Q^{2}\right)$ values are accessed via the PDF interface methods PDF : : $\operatorname{xfxQ}(\ldots)$ and PDF $:: \operatorname{xfxQ} 2(\ldots)$-the only distinction between these name variants is whether the scale argument is provided as an energy or energy-squared quantity. The most efficient way is the $Q^{2}$ argument, since this is the internal representation - it is more efficient to square a $Q$ argument than to square-root a $Q^{2}$ one. Overloadings of these functions' argument lists allow PDF values to be retrieved from the library either for a single flavour at a time, for all flavours simultaneously as a int $\rightarrow$ double std : : map, or for the standard QCD partons as a (pre-existing) std: : vector of doubles. Parton flavours not explicitly declared in a PDF object will return $x f\left(x ; Q^{2}\right)=0$.

\subsection{PDF metadata}

A key feature in the LHAPDF 6 design is a powerful "cascading metadata" system, whereby any information (integer, floating point, string, or homogeneous lists of them) can be attached to a PDF, a PDF Set, or the global configuration of the LHAPDF system via a string-valued lookup key. Access to metadata is via the general Info class, which is used directly for the global LHAPDF system configuration and specialised into the PDFSet and PDFInfo classes for set-level and PDFlevel metadata respectively.

Much of the physics content of LHAPDF is in fact encoded via the metadata system. For example, the value of $\alpha_{S}\left(M_{Z}\right)$ is defined via metadata: if it is not defined on a PDF, the system will automatically fall back to looking in the containing PDF Set, and finally the LHAPDF configuration for a value before throwing an error (or accepting a user-supplied default). The metadata information is set in the PDF/PDF set/global configuration data files, as described later, and any metadata key may be specified at any level (with more specific levels overriding more generic ones). The main motivation for the cascade is reduced duplication and easier configuration: a global change in behaviour need not be set in every PDF, and set-level information need not be duplicated in the data files for every one of its members. All metadata values set from file may also be explicitly overridden in the user code.

\subsection{Object and memory management}

A very important change in LHAPDF6 with respect to v5 is how the user manages the memory associated with PDFs - namely that they are now fully responsible for it.
A user may create as many or as few PDFs at runtime as they wish - there is neither a necessity to create a whole set at a time, nor any need to re-initialise objects, nor a limitation to NMXSET concurrent PDF sets. The flip-side to this flexibility is that the user is also responsible for cleaning up this memory use afterwards, either with manual calls to delete or by use of e.g. smart pointers.

Many objects, including PDFs, are created in factory functions such as LHAPDF $:: \operatorname{mkPDF}(\ldots)$, LHAPDF $:$ : getPDFSet (...), and LHAPDF : : PDFSet : :mkPDFS (). Internally these functions typically call the new operator so that the memory is allocated on the heap and outlives the scope of the calling function. We use a naming convention to indicate when the user needs to delete the created objects: if the function name starts with "mk", then the return type will be pointer(s) and the user is responsible for deletion. Note that LHAPDF : : getPDFSet $(\ldots)$ is not such a function: PDFSet is a lightweight object shared between the set members and hence its memory is automatically managed and is only exposed to the user via a reference handle, not a pointer.

Creation of PDFs is usually done via the factory functions LHAPDF : :mkPDF $(\ldots)$ and LHAPDF $:: \operatorname{mkPDFs}(\ldots)$, which take several forms of argument list. mkPDF, which returns a heap-allocated $\mathrm{PDF}^{*}$, either takes two identifier arguments the string name of the PDF set, plus the integer PDF member offset within the set - or a single string which encodes both properties with a slash separator, e.g. mkPDF ( "CT10nlo/0") to refer to the central member of the CT10nlo set. For convenience, if the $/ 0$ is omitted when specifying a single PDF, the first (nominal) member is taken as implied. This string-based lookup is extremely convenient ${ }^{2}$ and we encourage uptake of this scheme as standard syntax for referencing individual PDF members. A final form takes a single integer argument, which gives the global LHAPDF ID code for the desired PDF set member. The mkPDFs (...) functions behave similarly, but only the set name is specified (or implied when calling LHAPDF : : PDF Set : : mkPDFs ( ) ). If no further argument is given, the PDFs are returned as a vector $\langle\mathrm{PDF} *\rangle$, but an extra argument of templated type vector $<\mathrm{T}>$ may also be given and will be filled in-place for better computational efficiency and to allow automatic use of smart pointers.

\subsection{PDF value calculation}

The PDF $x f\left(x ; Q^{2}\right)$ values may come from any implementation, derived from the abstract PDF class, although (reflecting the reality of real-world PDF usage) the only current provider is the GridPDF class which provides PDF values interpolated from data files.

\footnotetext{
${ }^{2}$ Extension of this scheme is anticipated for PDFs with nuclear correction factors in a future release.
} 
These data files consist of PDF values for each flavour evaluated on a rectangular grid of "knots" in $\left(x, Q^{2}\right)$, with values for all flavours given at each point. The spacing of the knot positions in $x$ and $Q^{2}$ is not prescribed, but the physical nature of PDFs means that most natural and efficient representation is to use uniform or near-uniform distributions in $\log x$ and $\log Q^{2}$.

In fact, each PDF may contain arbitrarily many distinct grids in $Q^{2}$, in order to allow for parton density discontinuities (or discontinuous gradients) across quark mass thresholds. This gives the possibility of correct handling of evolution discontinuities in NNLO PDFs, and is used by the MSTW2008 and NNPDF3.0 fits. There is no requirement that the subgrid boundaries lie on quark masses - they may be treated as more general thresholds if wished. The $Q^{2}$ boundaries of these subgrids, and the $x, Q^{2}$ knots within them must be the same for all flavours in the PDF. The mechanisms for efficient lookup from an arbitrary $\left(x, Q^{2}\right)$ to the containing subgrid, and of the surrounding knots within that subgrid (and of specific flavours at each point) are implemented in the GridPDF class and associated helper structures.

Since several applications of PDFs, notably their use in Monte Carlo parton shower programs, require a probabilistic interpretation of the PDF values, a "force positive" option has been implemented to ensure (if requested) that negative $x f\left(x ; Q^{2}\right)$ values are not returned, either from actual negative values at interpolation knots or by a vagary of the interpolation algorithm. This is necessary for leadingorder or leading-log applications such as parton showers, but not in the matrix element computation of NLO showermatched generators. The force-positive behaviour is set via the Forcepositive metadata key, which takes values of 0 , 1 , or 2 to, respectively, indicate no forcing, forcing negative values to 0 , or forcing negative-or-zero values to a very small positive constant.

The interpolation of gridded PDF values to arbitrary points within the grid $x$ and $Q^{2}$ ranges is performed by a flexible system of interpolator objects.

\subsubsection{Interpolator system}

There are many possible schemes for PDF interpolation. To strike a balance between efficiency and complexity, we have implemented an interpolation based on cubic Hermite splines in $\log Q^{2}-\log x$ space as the default interpolation scheme in LHAPDF 6, implemented in the LogBicubicInter polator class, which inherits from an abstract Inter polator type.

Internally, the log-cubic PDF querying is natively done via $Q^{2}$ rather than $Q$, since event generator shower evolution naturally occurs in a squared energy (or $p_{\perp}$ ) variable and it is advisable to minimise expensive calls of sqrt. For this log-based interpolation measure, the logarithms of (squared) knot positions are pre-computed in the interpolator construction to avoid excessive log calls in calls to the interpolation function. In the regions close to the edges of each subgrid, where fewer than the minimum number of knots required for cubic spline interpolation are available, the interpolator switches automatically to linear interpolation.

This interpolation scheme is not hard-coded but is simply the standard value, "logcubic", of the Interpolator metadata key. This key is read at runtime when a PDF's value is first queried, and is used as the argument to a factory function whose job is to return an object implementing the Interpolator interface. If an alternative value is specified in the PDF set's . info file, in a specific member's . dat file, or is overridden by a call to PDF : : setInterpolator (...) before the PDF is first queried, then the corresponding interpolator will be used instead. At present, however, the alternative interpolators such as "linear" are intended more for debugging (and for edge-case fallbacks) than for serious physics purposes.

As the interpolator algorithm is runtime-configurable, there is the possibility of evolving better interpolators in a controlled way without changing previous PDF behaviours. So far there has been little incentive to do so, as specific problem regions like high- $x$ where uniform spacing of anchor points in $\log x$ becomes sub-optimal are most easily dealt with by locally increased knot density rather than a global increase in the complexity (and computational cost) of the interpolation measure.

Interpolation as described here only applies within the limiting ranges of the $\left(x, Q^{2}\right)$ grid (given by XMin-XMax and QMin-QMax metadata keys and accessed most conveniently via the $\mathrm{PDF}: \mathrm{xMin}($ ) etc. methods). Outside this range, a similar extrapolator system is used.

\subsubsection{Extrapolation system}

The majority of PDF interpolation codes included in LHAPDF 5 did not return a sensible extrapolation outside the limits of the grid, with many codes even returning nonsensical PDF values. Hence the default LHAPDF 5 behaviour was to "freeze" the PDFs at the boundaries, although this option could be overridden for the few PDF sets that did return sensible behaviour beyond the grid limits.

In particular, the MSTW interpolation code included in LHAPDF 5 made an effort to provide a sensible extrapolation to small- $x$, low- $Q$ and high- $Q$ values. A continuation to small $x$ values was performed by linearly extrapolating from the two smallest $\log x$ knots either the value of $\log x f$, if $x f$ was sufficiently positive, or just $x f$ itself otherwise. A similar continuation to high $Q$ values was performed based on linear extrapolation from the two highest $\log Q^{2}$ knots. Extrapolation to low $Q$ values is more ambiguous, but the choice made was to interpolate the anomalous dimension, 
$\gamma\left(Q^{2}\right)=\partial \log x f / \partial \log Q^{2}$, between the value at $Q_{\min }$ and a value of 1 for $Q \ll Q_{\min }$, so that the PDFs for $Q \sim Q_{\text {min }}$ behave as:

$x f\left(x ; Q^{2}\right)=x f\left(x ; Q_{\min }^{2}\right)\left(Q^{2} / Q_{\min }^{2}\right)^{\gamma\left(Q_{\min }^{2}\right)}$,

while for $Q \ll Q_{\min }$ the PDFs vanish as $Q^{2} \rightarrow 0$ like:

$x f\left(x ; Q^{2}\right)=x f\left(x ; Q_{\min }^{2}\right)\left(Q^{2} / Q_{\min }^{2}\right)$.

In LHAPDF $6,\left(x, Q^{2}\right)$ points outside the grid range trigger the same sort of function-object lookup as for in-range interpolation, but the returned object now implements the Extrapolator interface.

The default extrapolation, as of LHAPDF version 6.1.5, is an implementation of the MSTW scheme for use with all PDF sets, named the "continuation" extrapolator. Alternatives are also available: a "nearest" extrapolator as in LHAPDF 5, which operates by identifying the nearest in-range point in the grid and then using the correct interpolator to return the value at that point via a pointer back to the GridPDF object; and an "error" extrapolator which simply throws an error if out-of-range PDF values are requested. Uncontrolled evolution outside the range is not an option for LHAPDF 6's interpolation grids.

\section{$3.5 \alpha_{\mathrm{S}}$ system}

Consistent $\alpha_{\mathrm{S}}$ evolution is key to correct PDF evolution and usage: programs which use PDFs in cross-section calculations should also ensure, at least within fixed-order perturbative QCD computations, that they use $\alpha_{\mathrm{S}}$ values consistent with those used in the PDF fit. LHAPDF 6 contains implementations of $\alpha_{\mathrm{S}}$ running via three methods: an analytic approximation, a numerical solution of the ODE, and a $1 \mathrm{D}$ cubic spline interpolation in $\log Q$. All three methods implement the LHAPDF : : Alphas interface.

The first two of these methods are defined within the $\overline{\mathrm{MS}}$ renormalization-scheme, and for consistency this scheme should also be used for interpolation values supplied to the spline interpolation. The analytic and ODE implementations are based on the outlines given in Ref. [14] using the result from Ref. [15] for $b_{3}$, the results from Ref. [16] for the QCD decoupling coefficients $c_{n}$, and the result from Ref. [17] for the analytic four-loop approximation. Flavour thresholds/masses, orders of QCD running, and fixed points/ $\Lambda_{\mathrm{QCD}}$ are all correctly handled in the analytic and ODE solvers, and subgrids are available in the interpolation.

The ODE solver approximates the $\alpha_{\mathrm{S}}$ running by numerically solving the renormalization group equation up to four- loop order using the input parameters $M_{Z}, \alpha_{S}\left(M_{Z}\right)$ :

$$
\begin{aligned}
\mu^{2} \frac{d \alpha_{\mathrm{S}}}{d \mu^{2}} & =\beta\left(\alpha_{\mathrm{S}}\right) \\
& =-\left(b_{0} \alpha_{\mathrm{S}}^{2}+b_{1} \alpha_{\mathrm{S}}^{3}+b_{2} \alpha_{\mathrm{S}}^{4}+b_{3} \alpha_{\mathrm{S}}^{5}+O\left(\alpha_{\mathrm{S}}^{6}\right)\right) .
\end{aligned}
$$

The decoupling at flavour thresholds where we go from $n_{f}$ to $n_{f}+1$ active flavours or vice versa is currently calculated using under the assumption the flavour threshold is at the heavy quark mass, a restriction which will shortly be relaxed to allow use of generalised thresholds:

$\alpha_{\mathrm{S}}^{\left(n_{f}+1\right)}(\mu)=\alpha_{\mathrm{S}}^{\left(n_{f}\right)}(\mu)\left(1+\sum_{n=2}^{\infty} c_{n}\left[\alpha_{\mathrm{S}}^{\left(n_{f}\right)}(\mu)\right]^{n}\right)$.

If a more involved calculation is required, we suggest linking LHAPDF6 to a dedicated $\alpha_{\mathrm{S}}$ library such as that described in Ref. [18]. This evolution is used to dynamically populate an interpolation grid which is used thereafter for performance reasons.

The analytic approximation is given by the following expression, again up to four-loop order:

$$
\begin{aligned}
\alpha_{\mathrm{S}}(\mu)= & \frac{1}{b_{0} t}\left(1-\frac{b_{1} \ln t}{b_{0}^{2} t}+\frac{b_{1}^{2}\left(\ln ^{2} t-\ln t-1\right)+b_{0} b_{2}}{b_{0}^{4} t^{2}}\right. \\
& \left.-\frac{b_{1}^{3}\left(\ln ^{3} t-\frac{5}{2} \ln ^{2} t-2 \ln t+\frac{1}{2}\right)+3 b_{0} b_{1} b_{2} \ln t-1 / 2 b_{0}^{2} b_{3}}{b_{0}^{6} t^{3}}\right),
\end{aligned}
$$

where $t=\ln \left(\mu^{2} / \Lambda_{\mathrm{QCD}}^{2}\right)$. Here $\Lambda_{\mathrm{QCD}}$ takes distinct values for different $n_{f}$, and these are required input parameters for the number of active flavours that are desired in the calculation. General flavour thresholds are possible with the analytic solver.

The interpolation option uses a set of $\alpha_{\mathrm{S}}$ values and their corresponding $Q$ knots, provided as metadata, to interpolate using a log-cubic interpolation with constant extrapolation for $Q^{2}>Q_{\text {last }}^{2}$ and logarithmic gradient extrapolation for $Q^{2}<Q_{\text {first }}^{2}$. Discontinuous subgrids are supported, to allow improved treatment of the impact of flavour thresholds on $\alpha_{\mathrm{S}}$ evolution.

These $\alpha_{S}$ evolution options are specified, cf. the grid interpolators and extrapolators, via an Alphas_Type metadata key on the PDF member or set. By default the general PDF quark mass, $M_{Z}$, etc. metadata parameters are used for $\alpha_{S}$ evaluation, but specific Alphas_* variants are also provided and take precedence. Other details of the $\alpha_{\mathrm{S}}$ scheme, such as variable or fixed flavour number scheme, are specified 
by the Alphas_FlavorScheme and Alphas_NumFlavors ${ }^{3}$ keys. Quark thresholds can be treated separately from the quark masses, but the latter are used as the default thresholds.

\section{Usage examples}

In this section we give brief demonstrations of how to acquire and use PDF objects in the three languages supported by LHAPDF 6: C++, Python, and Fortran (the latter via a legacy compatibility layer which provides the LHAPDF5 Fortran API, as will be described in Sect. 8).

\subsection{C++ example}

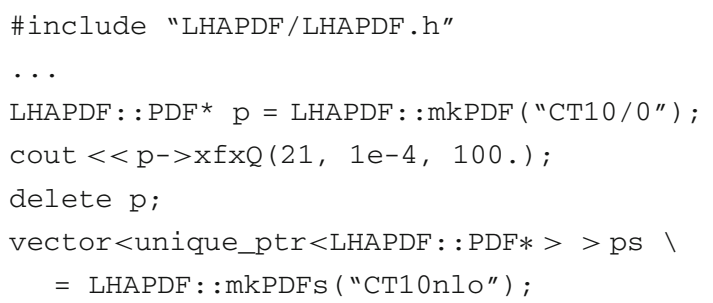

\subsection{Python example}

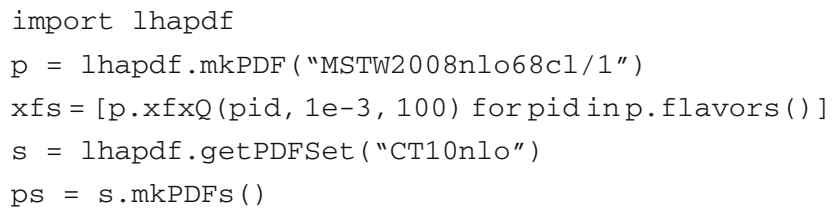

\subsection{Fortran example (same as for LHAPDF 5)}



\section{Data formats}

LHAPDF 6 uses a single system of metadata for all PDF sets, and a unified interpolation grid format for all PDFs implemented via the GridPDF class - this is the case for all currently active PDFs, both all those migrated from LHAPDF 5 and the several new sets supplied directly to LHAPDF6.

\footnotetext{
${ }^{3}$ Note that American spelling is used consistently in the LHAPDF 6 interface.
}

All these data files, and an index file used to look up PDF members by a unique global integer code - the LHAPDF ID, following the scheme started by PDFLIB - are searched for in paths which may be set via the code interface, which falls back to the \$LHAPDF_DATA_PATH environment variable if set, then to the legacy \$LHAPATH variable if set, and finally to the build-time $\langle$ install-prefix / share/LHAPDF / data directory. The search paths set via the API and via the environment variables may contain several different locations, separated in the usual way by colon ( : ) characters in the variables; as usual these are searched in left-to-right order, returning as soon as a match is found.

Since it is shared between all prospective PDF implementations and can influence the interpretation of the PDF data formats, we first describe the metadata format in some detail, then the data format for LHAPDF 6's standard interpolation grids.

\subsection{Metadata format}

Metadata is encoded in LHAPDF 6 using the standard YAML [19] syntax, and a uniform system is used for controlling system behaviours and storing PDF physical information. YAML is a simple data structure syntax designed as a more human readable/writeable variant of XML. Its use in LHAPDF 6 consists of dictionaries of key-value pairs, written as Key: Value. The LHAPDF keys are all character strings; the value types may be booleans, strings, integers, floating point numbers, or lists of numbers written as $[1,2,3 \ldots]$. Valid boolean values include true, false, yes, no, 1,0 , and capitalised variants. The yaml-cpp package [20] is embedded inside the LHAPDF library ${ }^{4}$ and is responsible for parsing of the YAML data sections, which are then available in $\mathrm{C}++$ typed fashion from the Info class and its specialisations.

Each PDF has a data file, the first part of which is YAML; these files share a set directory with a $\langle$ setname $\rangle$. info file which is in the same format; and lastly the global configuration lives in a lhapdf. conf file, again in YAML.

As already mentioned, metadata keys set at a more specific level will override those set more globally; it can hence be most efficient (for maintenance) to set a not-quite ubiquitous key at PDFSet level and override it in the minority of PDF members to which it does not apply. Major metadata keys and their types are listed in Table 1.

\subsubsection{System-level metadata}

The basic system-level configuration is set by a collection of metadata keys in the file lhapdf . conf - specifically the first file of that name to be found in the runtime search path, as is

\footnotetext{
${ }^{4}$ With a modified namespace to avoid clashes with external usage.
} 
Table 1 Main metadata keys used in LHAPDF6 along with their data types and descriptions. Full information on the standard metadata keys and their usage is found in the CONFIGFLAGS file in the LHAPDF code distribution, and on the LHAPDF website

\begin{tabular}{|c|c|c|c|}
\hline Name & Type & Default value & Description \\
\hline \multicolumn{4}{|l|}{ USUALLY SYSTEM- LEVEL } \\
\hline Verbosity & int & 1 & Level of information/debug printouts \\
\hline Pythia6LambdaV5Compat & bool & True & Return incorrect $\Lambda_{\mathrm{QCD}}$ values in the PYTHIA6 interface \\
\hline \multicolumn{4}{|l|}{ USUALLY SET- LEVEL } \\
\hline SetDesc & str & & Human-readable short description of the PDF set \\
\hline SetIndex & int & & Global LHAPDF/PDFLIB PDF set ID code of first member \\
\hline Authors & str & & Authorship of this PDF set \\
\hline Reference & str & & Paper reference(s) describing the fitting of this PDF set \\
\hline DataVersion & int & -1 & Version number of this data, to detect $\&$ update old versions \\
\hline NumMembers & int & & Number of members in the set, including central (0) \\
\hline Particle & int & 2,212 & PDG ID code of the represented composite particle \\
\hline Flavors & list[int] & & List of PDG ID codes of constituent partons in this PDF \\
\hline OrderQCD & int & & Number of QCD loops in calculation of PDF evolution \\
\hline FlavorScheme & str & & Scheme for treatment of heavy flavour (fixed/variable) \\
\hline NumFlavors & int & & Maximum number of active flavours \\
\hline MZ & real & 91.1876 & $Z$ boson mass in $\mathrm{GeV}$ \\
\hline MUp, ..., MBottom, MTop & real & $0.002, \ldots, 4.19,172.9$ & Quark masses in GeV \\
\hline Interpolator & str & Logcubic & Factory argument for interpolator making \\
\hline Extrapolator & str & Continuation & Factory argument for extrapolator making \\
\hline ForcePositive & int & 0 & Allow negative (0), zero (1), or only positive (2) $x f$ values \\
\hline ErrorType & str & & Type of error set (hessian/symmhessian/replicas/unknown) \\
\hline ErrorConfLevel & real & $68.268949 \ldots$ & Confidence level of error set, in percent \\
\hline XMin, XMax & real & & Boundaries of PDF set validity in $x$ \\
\hline QMin, QMax & real & & Boundaries of PDF set validity in $Q$ \\
\hline Alphas_Type & str & Analytic & Factory argument for $\alpha_{\mathrm{S}}$ calculator making \\
\hline AlphaS_MZ & real & 91.1876 & $Z$ boson mass in $\mathrm{GeV}$, for $\alpha_{\mathrm{S}}\left(M_{Z}\right)$ treatment \\
\hline Alphas_OrderQCD & int & & Number of QCD loops in calculation of $\alpha_{\mathrm{S}}$ evolution \\
\hline Alphas_Qs,_Vals & list[real] & & Lists of $Q \& \alpha_{\mathrm{S}}$ interpolation knots \\
\hline Alphas_Lambda4/5 & real & & Values of $\Lambda_{\mathrm{QCD}}^{(4)}$ and $\Lambda_{\mathrm{QCD}}^{(5)}$ for analytic $\alpha_{\mathrm{S}}$ \\
\hline \multicolumn{4}{|l|}{ USUALLY MEMBER- LEVEL } \\
\hline PafType & str & & Type of PDF member (central/error/replica) \\
\hline Format & str & & Format of data grid (1hagrid1/...) \\
\hline
\end{tabular}

the case for all file lookup in LHAPDF6. The system-level metadata can be obtained by loading the generic info object using the LHAPDF : : getConfig ( ) function.

The default set of such keys is relatively small and sets some uncontroversial values such as use of the log-cubic interpolator and the continuation extrapolator, and default quark and $Z$ boson masses.

The Verbosity key is also set here: this integer-valued parameter controls the level of output written to the terminal on loading PDFs and performing other operations, and by default is set to 1 , which produces a small announcement on first loading a PDF set; by comparison 0 is silent and 2 produces more detailed and more frequent print-outs.

\subsubsection{Set-level metadata}

As opposed to LHAPDF 5, where each PDF set was encoded in a single text data file, the LHAPDF 6 format is that each set is a directory with the same name as the set, which contains one $\langle$ setname $\rangle$. info file, plus the memberspecific data files. The common set-level metadata should be set in the .info file. The set-level metadata can be obtained by loading the lightweight PDFSet object using the LHAPDF : : getPDFSet ( ) function.

The bulk of metadata should be declared at the PDF set level, except in those sets where each member has a systematic variation in the information set via metadata keys such as 
quark masses/thresholds and $\alpha_{\mathrm{S}}$. The information typically specified at the set-level includes quark and $Z$ masses (even if the system-level defaults are appropriate, it is safest to repeat the values used for future-proofing), the PDG ID code of the parent particle (to allow for identifiable nuclear PDFs in future), and the error treatment, confidence level, etc. of the systematic uncertainty variations in the set, to permit automated error computation such as that described in Sect. 6.

\subsubsection{Member-level metadata}

As will be described in more detail below, in addition to the .info file in each PDF set directory, there is one ".dat" file for each PDF member in the set. This structure permits much faster lookup of set-level metadata and random access to single members in the set, compared to the one-file-per-set structure used by LHAPDF 5 .

The top section of each . dat file is devoted to memberlevel metadata in the usual format. This should contain the Format metadata key which will be used to determine what sort of PDF is being loaded and trigger the appropriate constructor (e.g. GridPDF, for key value lhagrid1) via a factory function to read the rest of the file. This header section ends with a mandatory line containing only three dash characters (---), the standard YAML sub-document separator. The PdfType key is also usually set here, to declare whether this member is a central or error/replica PDF member. Any other metadata key may also be declared at member-level, possibly overriding set-level values; this is particularly the case for special quark mass or $\alpha_{\mathrm{S}}$ systematic variation sets.

PDF member-level metadata can be loaded without needing to load the much larger data block by use of the LHAPDF : :mkPDFInfo ( . . ) factory functions.

\subsection{PDF grid data format}

Within the $\langle$ setname $\rangle$ directory, each PDF member has its own file named $\langle$ setname $\rangle\langle\langle n n n n\rangle$.dat, where $\langle n n n n\rangle$ is a 4-digit zero-padded representation of the member number within the set - for example member 0 is " 0000 " and member 51 is " 0051 " - reasonably assuming that there will be no need for PDF sets with more than 10,000 members. The "central" PDF set member must always be number 0 .

The splitting of PDF set data into one file per member permits faster random access to individual members (the central member being the most common), and permits an extreme space optimisation for circumstances which require it: PDF data directories may be cut down to only contain the subset of members which are going to be used. While not generally recommended, this may give a significant space saving and be useful for resource-constrained applications - for example, to allow LHC experiments' Grid installations to contain the central members of many PDF sets where distribution of the full sets would make unreasonable demands on Grid sites and kit distribution.

As already described, the first section in each . dat file contains a YAML header of member-specific metadata, until the --- separator line. After this line, the grid data begins. Each subgrid in $Q$ is treated separately and should be listed in the file in order of increasing $Q$ bin, separated again by --- separator lines. The file must be terminated by such a line after the last subgrid data block.

Within each subgrid block there is a three-line header then a large number of lines giving the PDF values at each $(x, Q)$ point. The first line in the header is a space-separated, ordered list of $x$ knot values; the second is a similar list of $Q$ knot values; and the third is a list of the particle ID codes to be given in the data block to follow. Note that although the interpolator/extrapolator implementations operate canonically in $Q^{2}$ (or $\log Q^{2}$ ) to avoid expensive square-root function calls in typical usage, in the data files we always use $Q$ to give the scale: this is for ease of interpretation and debugging, since physicists find it more natural to interpret scales related to e.g. the masses or transverse momenta of produced particles than the squares of such quantities. The particle codes listed on the third header line are in the standard PDG ID scheme, and must be given in the order that columns of PDF values will be presented in the remainder of the subgrid block. It is anticipated that the "generator specific" range of PDG ID codes may be used in future to permit valence/sea decompositions or aliasing of PDF components in the LHAPDF data files, but there has not yet been demand for such features.

The gridded PDF value data comes next, with each line giving an $x f(x ; Q)$ value for each of the parton ID codes given in the final line of the block header. The order of lines corresponds to a nested pair of loops over the $x$ and $Q$ knot lists given in the block header, e.g. what would result from the pseudocode

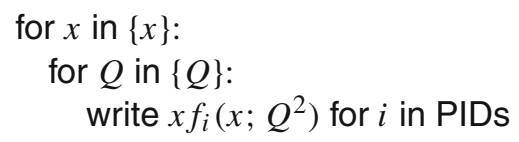

The lines hence come in groups of lines with fixed $x$, each group containing as many lines as there are $Q$ knots, with the total subgrid containing $|\{x\}| \times|\{Q\}|$ lines of $x f$ grid data in addition to the three header lines that specify the knot positions and parton flavours. The GridPDF parser makes many consistency checks on the correctness of the format.

\section{$5.3 \alpha_{\mathrm{S}}$ interpolation data format}

If the interpolation scheme is used for getting $\alpha_{\mathrm{S}}$ values from a PDF (Alphas_Type $=$ ipol), the interpolation knot $\alpha_{S}$ values and $Q$ positions are given as lists of floating point values for the metadata keys Alphas_Vals and AlphaS_Qs respectively. These are used for log-cubic interpolation in the 
usual way. Naturally the two lists must be of the same length. Subgrid boundaries in $Q$ are expressed by a repetition of the boundary $Q$ value - the corresponding $\alpha_{\mathrm{S}}$ values should be given as the $\alpha_{\mathrm{S}}$ limits from below and above the boundary.

\subsection{Index file}

The LHAPDF::mkPDF(int), LHAPDF::lookupPDF(int), and LHAPDF: : lookupLHAPDFID (string, int) factory functions make use of the global LHAPDF ID code and its mapping to PDF members. This mapping is done via the pdfsets . index file, which must be found in the search paths for these lookup functions to work. This file contains three data columns separated by whitespace: the LHAPDF ID, the set name, and the set's latest data version. The only entries in the index file are the first entries in each PDF set, since the ID codes and containing sets of any member may be extracted from these.

The LHAPDF ID index codes are given in each PDF set . info file via the Set Index metadata key, which gives the LHAPDF ID number of the first (central) member in the set. To ease maintenance work and minimise errors, the index file is generated automatically by loading and querying the . info files from all the PDF sets. LHAPDF's online documentation of available PDF sets is also generated by this method.

\subsection{Distribution and updating}

LHAPDF 6 breaks the tight binding of PDF data files and the LHAPDF code library: releases of new PDF set data now happens in general out of phase with software releases, permitting much faster release of PDF sets for use via LHAPDF. This was a major design goal of LHAPDF 6.

The sets are distributed as $\langle$ setname $\rangle$.tar.gz archive files, each one expanding to the $\langle$ setname $\rangle$ directory which contains the set's metadata (. info) and data (. dat) files. A typical PDF set with 50 members and 5 quark flavours corresponds to a 5-10 MB compressed tarball, which on expansion will occupy 20-30 MB. The 100-member NNPDF sets, which also include top (anti)quark PDFs, are somewhat larger at $\mathcal{O}(30 \mathrm{MB})$ compressed and $\mathcal{O}(80 \mathrm{MB})$ expanded; sets with fewer members or fewer flavours require correspondingly less disk space. Directly using the unexpanded tarballs is not supported, but a trick to do so will be described in Sect. 9 .

The only update required for full usability of a new PDF set is an updated version of the pdfsets. index file, although this is only needed for PDF use via the LHAPDF ID code: access to PDFs by set-name + set-member number does not use the index file and is encouraged for robustness and human readability. New official PDF set data will be uploaded to the LHAPDF website [21] along with an updated, automatically generated version of the pdfsets. index file. Official PDF sets will also be distributed, both tarballed and expanded, via the CERN AFS and CVMFS distributed file systems.

Officially supported PDF sets must contain the DataVersion integer metadata key to allow for tracking of bugfix releases of the set data files. The latest such number is written into the pdfsets.index file, and can be used to detect when an update is available for a PDF set installed on a user's system. LHAPDF6 provides and installs a PDF data management script simply called lhapdf, with an interface similar to the Debian/Ubuntu Linux apt-get command: calling lhapdf list and lhapdf install will respectively list and install PDFs from the Web, lhapdf update will download the latest index file from the LHAPDF website, and lhapdf upgrade will download updated versions of PDF set files if notified as available in the current index file. The rest of the script features are interactively documented by calling lhapdf --help.

In future PDF sets may be released which require LHAPDF features such as newer grid formats, which are only available after a particular LHAPDF release. In this situation, which has not yet been encountered, the set should declare the MinLHAPDFVersion metadata flag to have an integer value corresponding to the earliest LHAPDF 6 version with which it is compatible. This integer version code will be described in Sect. 8.

\section{PDF uncertainties}

Over the last decade or so, it has become standard practice for PDF fits to propagate the experimental uncertainties on the fitted data points and provide a number of alternative PDF members in addition to the central member. An estimate of PDF uncertainties on either the PDFs themselves, or derived quantities such as parton luminosities or crosssections, can then easily be calculated with a simple formula using the quantity calculated for all members of the PDF set. Correlations between two quantities can also be calculated, for example, to establish the sensitivity of a particular crosssection to a PDF of a particular flavour. However, in practice, there are multiple formulae in common use depending on the PDF set together with a variety of different confidence levels, requiring some specialist knowledge from the user in order to apply the correct formula, and potentially leading to mistakes by non-experts that could severely underestimate or overestimate the importance of PDF uncertainties. Moreover, each user or code that calculates PDF uncertainties needs to implement the correct formula for each PDF set and possibly rescale uncertainties to a desired confidence level, typically with branching based on the name of the PDF set, resulting in a vast duplication of effort.

Starting from LHAPDF 5.8.8 first steps were taken towards a more automatic calculation of PDF uncertain- 
ties by providing Fortran subroutines GetPDFUncType, GetPDFuncertainty and GetPDFcorrelation that would attempt to use the appropriate formulae based on the name of the grid format. However, $\mathrm{C}++$ versions of these functions were not implemented and it was not straightforward to discern the confidence level of a given PDF set. Starting from LHAPDF 6.1.0 member functions were implemented in the PDFSet class making use of the new set-level metadata, specifically ErrorType and ErrorConfLevel, with several extensions to the original Fortran subroutines. Here we describe these functions and the formulae implemented based on the chosen PDF set, for each of the three currently supported values of ErrorType, namely hessian, symmhessian or replicas. ${ }^{5}$ An example program (testpdfunc.cc) demonstrates the basic functionality. See, for example, Section 2.2.3 of Ref. [4] for a more comprehensive review of the different approaches, and Refs. [22,23] for more discussion of the relevant formulae.

\section{1 set.uncertainty(values, cl, alternative)}

This function takes as input a vector of values and returns a PDFUncertainty structure containing a central value, asymmetric (errplus and errminus) and symmetric (errsymm) uncertainties, and the scale factor used to rescale uncertainties to the desired confidence level ( $\mathrm{cl}$, in percent), by default 1 -sigma $=\operatorname{erf}(1 / \sqrt{2}) \simeq 68.268949 \%$. The formulae used for the calculation depend on the value of ErrorType and are hidden from the user, but for reference we give the different formulae below for each ErrorType. The alternative option is only relevant for the replicas case.

hessian: Given a central PDF member $S_{0}$ and $2 N_{\text {par }}$ eigenvector PDF members $S_{i}^{ \pm}\left(i=1, \ldots, N_{\mathrm{par}}\right)$, where $N_{\text {par }}$ is the number of fitted parameters, the central value $F_{0}$ and asymmetric $\left(\sigma_{F}^{ \pm}\right)$or symmetric $\left(\sigma_{F}\right)$ PDF uncertainties on a PDF-dependent quantity $F(S)$ are given by:

$$
\begin{aligned}
F_{0} & =F\left(S_{0}\right), \quad F_{i}^{+}=F\left(S_{i}^{+}\right), \quad F_{i}^{-}=F\left(S_{i}^{-}\right), \\
\sigma_{F}^{+} & =\sqrt{\sum_{i=1}^{N_{\mathrm{par}}}\left[\max \left(F_{i}^{+}-F_{0}, F_{i}^{-}-F_{0}, 0\right)\right]^{2},} \\
\sigma_{F}^{-} & =\sqrt{\sum_{i=1}^{N_{\mathrm{par}}}\left[\max \left(F_{0}-F_{i}^{+}, F_{0}-F_{i}^{-}, 0\right)\right]^{2},}
\end{aligned}
$$

\footnotetext{
5 The more complicated prescription for the HERAPDF/ATLAS “VAR" model and parametrisation errors differs between the different sets and is not currently supported.
}

$\sigma_{F}=\frac{1}{2} \sqrt{\sum_{i=1}^{N_{\mathrm{par}}}\left(F_{i}^{+}-F_{i}^{-}\right)^{2}}$.

symmhessian: For the simpler case where only a central PDF member $S_{0}$ and $N_{\text {par }}$ eigenvector PDF members $S_{i}$ $\left(i=1, \ldots, N_{\mathrm{par}}\right)$ are provided, the central value and PDF uncertainties are calculated as:

$F_{0}=F\left(S_{0}\right), \quad F_{i}=F\left(S_{i}\right)$,
$\sigma_{F}^{+}=\sigma_{F}^{-}=\sigma_{F}=\sqrt{\sum_{i=1}^{N_{\mathrm{par}}}\left(F_{i}-F_{0}\right)^{2} .}$

replicas: Given a set of $N_{\text {rep }}$ equiprobable Monte Carlo replica PDF members $\mathcal{S}^{k}\left(k=1, \ldots, N_{\text {rep }}\right)$, created either by making fits to randomly shifted data points or by randomly sampling the parameter space, the central value and PDF uncertainties are by default (alternative=false) given by the average and standard deviation over the replica sample:

$$
\begin{aligned}
& F_{0}=\langle F\rangle=\frac{1}{N_{\text {rep }}} \sum_{k=1}^{N_{\text {rep }}} F\left(\mathcal{S}^{k}\right), \\
& \sigma_{F}^{+}=\sigma_{F}^{-}=\sigma_{F}=\sqrt{\frac{1}{N_{\text {rep }}-1} \sum_{k=1}^{N_{\text {rep }}}\left[F\left(\mathcal{S}^{k}\right)-F_{0}\right]^{2}} \\
& =\sqrt{\frac{N_{\text {rep }}}{N_{\text {rep }}-1}\left[\left\langle F^{2}\right\rangle-\langle F\rangle^{2}\right] .}
\end{aligned}
$$

Alternatively (if al ternative=true), a confidence interval (with level $\mathrm{cl}$ ) is constructed from the probability distribution of replicas, with the central value $F_{0}$ given by the median, then the interval $\left[F_{0}-\sigma_{F}^{-}, F_{0}+\sigma_{F}^{+}\right]$contains $\mathrm{c} 1 \%$ of replicas, while the symmetric uncertainty is simply defined as $\sigma_{F}=\left(\sigma_{F}^{+}+\sigma_{F}^{-}\right) / 2$.

\section{2 set.correlation(valuesA, valuesB)}

This function takes as input two vectors valuesA and valuesB, containing values for two quantities $A$ and $B$ computed using all PDF members, and returns the correlation cosine $\cos \phi_{A B} \in[-1,1]$. Values of $\cos \phi_{A B} \approx 1$ mean that $A$ and $B$ are highly correlated, values of $\approx-1$ mean that they are highly anticorrelated, while values of $\approx 0$ mean that they are uncorrelated. Again, we give the different formulae below for each ErrorType, although these formulae are invisible to the user. 
hessian: The correlation cosine is calculated as:

$\cos \phi_{A B}=\frac{1}{4 \sigma_{A} \sigma_{B}} \sum_{i=1}^{N_{\mathrm{par}}}\left(A_{i}^{+}-A_{i}^{-}\right)\left(B_{i}^{+}-B_{i}^{-}\right)$,

where the uncertainties $\sigma_{A}$ and $\sigma_{B}$ are calculated using the symmetric formula, Eq. (13).

symmhessian: Similarly, the correlation cosine is:

$\cos \phi_{A B}=\frac{1}{\sigma_{A} \sigma_{B}} \sum_{i=1}^{N_{\mathrm{par}}}\left(A_{i}-A_{0}\right)\left(B_{i}-B_{0}\right)$.

replicas: In the Monte Carlo approach:

$\cos \phi_{A B}=\frac{N_{\text {rep }}}{N_{\text {rep }}-1} \frac{\langle A B\rangle-\langle A\rangle\langle B\rangle}{\sigma_{A} \sigma_{B}}$,

where the average $\langle A\rangle$ and standard deviation $\sigma_{A}$ are defined in Eqs. (16) and (17), respectively.

\section{3 set.randomValueFromHessian (values, randoms, symmetrise)}

This function will generate a random value from a vector of values, containing values for a quantity $F$ computed using all PDF members of a hessian (or symmhessian) PDF set, and a vector of random numbers randoms sampled from a Gaussian distribution with mean zero and variance one. Random values generated in this way [23] can subsequently be used for applications such as Bayesian reweighting [24-26] or combining predictions from different PDF fitting groups (as an alternative to taking the envelope) [4]. Below we give the formulae used for each relevant ErrorType.

hessian: For the option symmetrise=false, we build a random value of a quantity $F$ according to:

$$
F^{k}=F\left(S_{0}\right)+\sum_{j=1}^{N_{\mathrm{par}}}\left[F\left(S_{j}^{ \pm}\right)-F\left(S_{0}\right)\right]\left|R_{j}^{k}\right|,
$$

where either $S_{j}^{+}$or $S_{j}^{-}$is chosen depending on the sign of the Gaussian random number $R_{j}^{k}$. We can repeat this procedure to generate $N_{\text {rep }}$ random values, where $k=1, \ldots, N_{\text {rep. }}$. However, this asymmetric prescription means that the average $\langle F\rangle$ over the $N_{\text {rep }}$ values does not tend to the best-fit $F\left(S_{0}\right)$ for large values of $N_{\text {rep }}$. Hence the default behaviour (symmetrise=true) is to use a symmetrised formula ensuring this condition: ${ }^{6}$

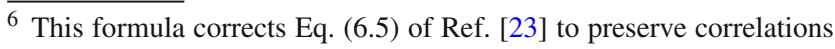
by not taking the absolute value of the quantity in square brackets.
}

$$
F^{k}=F\left(S_{0}\right)+\frac{1}{2} \sum_{j=1}^{N_{\mathrm{par}}}\left[F\left(S_{j}^{+}\right)-F\left(S_{j}^{-}\right)\right] R_{j}^{k} .
$$

symmhessian: In this case the symmetrise option has no effect and the formula is:

$$
F^{k}=F\left(S_{0}\right)+\sum_{j=1}^{N_{\mathrm{par}}}\left[F\left(S_{j}\right)-F\left(S_{0}\right)\right] R_{j}^{k} .
$$

An example program (hessian2replicas.cc) is provided that uses the randomValueFromHessian function to convert an entire hessian (or symmhessian) PDF set into a corresponding PDF set of Monte Carlo replicas.

\section{PDF reweighting}

A common use of PDFs is reweighting of event samples to behave as if they had originally been generated with PDFs other than the one that was actually used. This is particularly an effective strategy when applying a PDF uncertainty procedure such as the PDF4LHC recommendation [27] which involves predictions from $\sim 200$ PDF members - generating 200 independent MC samples is unrealistic and hence reweighting is a common approach. The reweighting factor for a leading-order hadron-hadron process from PDF $x f\left(x ; Q^{2}\right)$ to PDF $x g\left(x ; Q^{2}\right)$ is defined as

$w=\frac{x_{1} g_{i / B 1}\left(x_{1} ; Q^{2}\right)}{x_{1} f_{i / B 1}\left(x_{1} ; Q^{2}\right)} \cdot \frac{x_{2} g_{j / B 2}\left(x_{2} ; Q^{2}\right)}{x_{2} f_{j / B 2}\left(x_{2} ; Q^{2}\right)}$.

But we must note limitations in this strategy: a single welldefined set of partonic initial conditions is only defined at tree level, where there are no real- and virtual-emission counterterms to deal with. Reweighting higher-order calculations where counter-terms are involved requires deeper knowledge of the event generation than is typically available to users who wish to retrospectively reweight an existing event sample - it is much more appropriately done by the NLO $\mathrm{MC}$ generator code itself, and this is supported by at least the Sherpa [28], POWHEG-BOX [29], and MadGraph5_aMC@NLO [30] generator packages.

Further limitations are that PDF reweighting is typically applied only at the fixed-order matrix element level. Partonshower-matched event simulations also include PDF terms in the Sudakov form factors that appear in initial-state radiation emission probabilities, and these should strictly also be reweighted - but doing so consistently would require a sum over possible emission histories, which has yet to be formalised or implemented in such programs. And finally there is the issue of $\alpha_{S}$ consistency: if reweighting PDFs then appearances of the strong coupling - ideally both in the 
matrix element and shower - should also be reweighted. As this tends not to be done, PDF reweighting should only be done between PDFs with similar $\alpha_{\mathrm{S}}$ values in the scale range of the process. In particular reweightings between LO and NLO PDFs, which tend to have very different $\alpha_{\mathrm{S}}$ values, are strongly discouraged.

LHAPDF 5 provided no built-in support for reweighting, since the operation in Eq. (24) is numerically trivial. However it has transpired that within experimental collaborations there was demand for a "tool" to assist with this calculation. In the interests of usability LHAPDF 6 hence provides helper functions for computation of reweighting factors, in the LHAPDF/Reweighting.h header file. These are divided into two categories - single-beam functions which calculate the individual weighting factors for each beam, and two-beam functions which multiply together the weights for the two beams. The single-beam function signature is LHAPDF : : weightxQ2 $(i, x, Q 2$, pdf_f, pdf_g, aschk=0.05), which will reweight $x f_{i}\left(x ; Q^{2}\right) \rightarrow$ $x g_{i}\left(x ; Q^{2}\right)$. The optional aschk argument gives a threshold for the relative difference in $\alpha_{\mathrm{S}}\left(Q^{2}\right)$ between the two PDFs before the LHAPDF system will print a warning: this tolerance may be set negative to disable checking, but this is not advised for physics reasons. The pdf_f, $g$ arguments to this function may be given either as (const) references to $\mathrm{PDF}$ objects or as any kind of (smart or raw) PDF pointer. The equivalent two-beam functions have the same form, only generalised to have two parton ID and two $x$ arguments.

\section{LHAPDF 5/PDFLIB compatibility}

Due to the ubiquity of LHAPDF as a source of PDF information in HEP software, it would be unrealistic to release LHAPDF 6 without also providing a route for this mass of pre-existing code to continue to work.

\subsection{Legacy code interfaces}

To this end, legacy interfaces have been provided to the Fortran LHAPDF and PDFLIB interfaces, and to the LHAPDF 5 $\mathrm{C}++$ interface. These are written in $\mathrm{C}++$, and following the naming used in LHAPDF 5 to denote the backward compatibility interface with PDFLIB, are called the "LHAGlue" interface. It is entirely localised to the LHAGlue.h and LHAGlue. cc files within LHAPDF6.

The Fortran compatibility interfaces are implemented in $\mathrm{C}++$ using extern " $\mathrm{C}$ " linkage and the GCC Fortran symbol mangling conventions. Since there is a mismatch between the unlimited, dynamic memory allocation model of LHAPDF6's native C++ interface and the static, preallocated slots model of LHAPDF 5, a state machine was implemented to manage $\mathrm{PDF}$ object creation and deletion in numbered slots via the Fortran LHAPDF 5 initpdfsetm and initpdfm routines. For simplicity many of the $\mathrm{C}++$ LHAPDF 5 API functions were implemented via calls to these Fortran state-machine functions to reproduce the LHAPDF 5 behaviour.

Since the data format has changed in LHAPDF 6 and there are no longer any data files with the LHAPDF 5 . LHpdf or .LHgrid file extensions, calls to initpdfsetm which specify a name with such an extension will simply have it stripped off before continuing with PDF loading. There is a special case of this for the CTEQ6L1 PDF [31], which was accidentally implemented in LHAPDF 5 with the misspelt name cteq611. LHpdf: this name will automatically be translated to the correct name, cteq611, by which it is called in LHAPDF 6.

The legacy interfaces also contain a special case behaviour in the reporting of $\Lambda_{\mathrm{QCD}}^{(4)}$ and $\Lambda_{\mathrm{QCD}}^{(5)}$, which never worked correctly for the LHAPDF 5 PDFLIB-type common-block interface to PYTHIA 6 [32]. This value reporting is fixed in LHAPDF 6, but in the meantime many tunes of PYTHIA 6's physics modelling have been built around the assumption that an invalid value would be reported and PYTHIA would default to 0.192, the $\Lambda_{\mathrm{QCD}}^{(4)}$ value of the CTEQ5L PDF [33]. Since PYTHIA 6 is itself now largely replaced by its successor, Pythia 8 [34], and it is important that many of these tunes continue to work with an implicitly incorrect $\Lambda_{\mathrm{QCD}}$ value, a boolean metadata key Pythia6LambdaV5Compat has been provided to trigger the old physically incorrect but practically convenient behaviour. This flag is set true by default in the system thapdf.conf file, and may be changed in this file or by runtime use of the metadata API.

\subsection{Version detection hooks}

As well as these compatibility interfaces, LHAPDF 6 provides mechanisms to allow $\mathrm{C}++$ applications which use LHAPDF 5 to detect which version they are compiling against and hence migrate smoothly to the new version. Three $\mathrm{C}++$ preprocessor macros are provided for this purpose:

LHAPDF_VERSION provides a string version of the 3integer release version tuple (cf. the current release 6.1.4); LHAPDF_VERSION_CODE is a version of this information encoded into a single integer by multiplying the first and second numbers by 10,000 and 100 respectively, then adding the three numbers together (making the 6.1.4 release have a single-integer code of 60104);

LHAPDF_MAJOR_VERSION is the first number in the version 3-tuple, as an integer (i.e. 6 for version 6.1.4).

These macros can be portably accessed by \#include'ing the LHAPDF / LHAPDF . $h$ header, which is available in both version 5 and version 6 , and the integer codes can be used as a preprocessor test to separate code for calling LHAPDF 5 
routines from the new, more powerful LHAPDF 6 ones, for example:

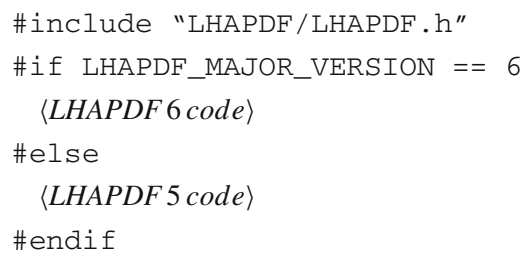

\subsection{Uptake and prospects}

The legacy interfaces have been successfully tested with a variety of widely-used MC generator codes, including PYTHIA 6 [32], HERWIG 6 [35], POWHEG-BOX [29], and aMC@NLO [30]. The main C++ parton shower generators, from Sherpa 2.0.0 [28], Herwig++ 2.7.1 [36], and Pythia 8.200 [34] onwards all support LHAPDF6 via the native C++ API. The global LHAPDF ID code is still in use and will continue to be allocated for submitted PDFs, meaning that the PDFLIB and LHAPDF 5 Fortran interfaces can continue to be used for some time, and will now return more correct values in some circumstances (e.g. $\alpha_{S}$ values in multi-set mode).

An improved Fortran interface to LHAPDF 6 is intended but has not yet progressed beyond initial stages; we welcome input from the Fortran MC generator community in particular on what features they would like to see.

\section{Benchmarking and performance}

The re-engineering of LHAPDF has impact upon the memory and CPU performance of the library. The main performance target in the redesign was to greatly reduce the multiple-GB static memory requirement of an LHAPDF 5 build with full multiset functionality. We describe the effect on this performance metric in the following section, and also mention the impact on CPU performance and data-file disk space requirements. We also describe some possible avenues for further performance improvements.

\subsection{Memory requirements}

The memory problems of LHAPDF 5 fundamentally stem from the Fortran 77 limitation to static memory allocation, and the use of large static arrays for PDF value interpolation in each PDF family's "wrapper" routine (i.e. the code which interfaced the native PDF group code into the LHAPDF 5 framework). By the time of LHAPDF 5.9.1, the proliferation of such wrapper routines meant that $2.04 \mathrm{~GB}$ of static memory was declared as required by the libLHAPDF library. This static memory requirement was incompatible with LHC
Table 2 Static memory requirements in $\mathrm{kB}$ for LHAPDF version 5 and 6 before any PDF allocation, broken down into the requirements for function, initialised data, and uninitialised data. LHAPDF 6 is much lighter on all counts, but the overwhelmingly most important number is the reduction in uninitialised data from more than $2 \mathrm{~GB}$ down to less than $1 \mathrm{MB}$. LHAPDF 6 memory only becomes substantial when PDF objects are created, and is proportional to the grid sizes of those PDFs

\begin{tabular}{lccr}
\hline Version & Functions & Init. data & Uninit. data \\
\hline 5.9 .1 & 1509.1 & 142.0 & $\mathbf{2 , 0 3 9 , 4 0 5 . 4}$ \\
6.1 .5 & 265.3 & 8.5 & $\mathbf{1 . 6}$ \\
\hline
\end{tabular}

computing systems, and the restricted memory builds used to work around process accounting limits were suitable only for the most basic sort of event generation; working around LHAPDF's technical limitations became a rite of passage in LHC data analysis.

The dynamic memory model in LHAPDF 6 completely solves this problem, as illustrated by the static memory information obtained by running the size command on the equivalent libraries between versions 5 and 6 of LHAPDF: this information is shown in Table 2 . All static memory requirements have been greatly reduced by the version 6 redesign, and the total static memory footprint is now just $280 \mathrm{kB}$, but the headline figure is the reduction in static uninitialised data size from more than $2 \mathrm{~GB}$ to a negligible $1.6 \mathrm{kB}$. This does not reflect the total memory requirements of LHAPDF 6 in active use - allocating a GridPDF will typically require a few hundred $\mathrm{kB}$, and loading a whole set into memory will require $\mathcal{O}(10 \mathrm{MB})$, but the user is now fully in control of when they allocate and deallocate that memory, as well as being able to load single PDF set members, an option not available in LHAPDF 5.

\subsection{CPU performance}

LHAPDF 6 was not specifically engineered for CPU performance gains, since this was not typically a severe issue with LHAPDF 5. However, particularly because of the approach taken to multiple parton-flavour evolution in GridPDF interpolation, there is some impact on CPU performance.

In LHAPDF 5 the performance was dependent on which PDF set was being used, as each wrapper routine was implemented independently and some were better optimised than others; however, the evolvePDF and $\mathrm{xfx}$ routines always returned a 13-element array of PDF values for the gluon + $2 \times 6$ quark flavours. They hence tended to be implemented such that the $x-Q^{2}$ "positional" part of the interpolation weights was only computed once, rather than being redundantly recomputed for every flavour at that point. This means that LHAPDF 6 interpolation is currently slightly slower than for LHAPDF 5 if all flavours are evaluated at every $\left(x, Q^{2}\right)$ point; however, if only one flavour is required at a phase 
Table 3 Times taken for phase space integration and CKKW-merged event generation using the Sherpa MC event generator with LHAPDF 5 $\left(t_{5}\right)$ and LHAPDF $6\left(t_{6}\right)$ via interface code optimised for each LHAPDF version, and the speed improvement ratio $t_{5} / t_{6}$. In all cases LHAPDF 6 runs faster than $\mathrm{v} 5$, in some (process- and PDF-specific) cases, faster by factors of $2-6$

\begin{tabular}{|c|c|c|c|}
\hline Process/PDF & $t_{5}$ & $t_{6}$ & $t_{5} / t_{6}$ \\
\hline \multicolumn{4}{|c|}{ Cross-section integrations, $1 \mathrm{M}$ phase space points } \\
\hline \multicolumn{4}{|l|}{ CT10 } \\
\hline$p p \rightarrow j j$ & $23^{\prime} 10^{\prime \prime}$ & 9'17”' & 2.5 \\
\hline$p p \rightarrow \ell \ell$ & 4’12”' & 2’02” & 2.1 \\
\hline$p p \rightarrow H(\mathrm{ggF})$ & $0 ’ 20 ”$ & $0 ’ 15 ”$ & 1.3 \\
\hline \multicolumn{4}{|l|}{ NNPDF23nlo } \\
\hline$p p \rightarrow j j$ & $54^{\prime} 40^{\prime \prime}$ & 9’28”' & 5.8 \\
\hline$p p \rightarrow \ell \ell$ & 8'06" & 2’33”' & 3.2 \\
\hline$p p \rightarrow H(\mathrm{ggF})$ & 0 '25”' & $0 ’ 11 ”$ & 2.3 \\
\hline \multicolumn{4}{|c|}{ CKKW event generation, 100k $p p \rightarrow \leq 4$ jet events } \\
\hline \multicolumn{4}{|c|}{ CT10 } \\
\hline Weighted & $43 ’ 02 ”$ & $35^{\prime} 47^{\prime \prime}$ & 1.2 \\
\hline Unweighted & 5 h04'39”' & $4 \mathrm{~h} 30$ '26”' & 1.1 \\
\hline \multicolumn{4}{|l|}{ NNPDF23nlo } \\
\hline Weighted & $47^{\prime} 47^{\prime \prime}$ & $27^{\prime} 20^{\prime \prime}$ & 1.7 \\
\hline Unweighted & $6 \mathrm{~h} 44^{\prime} 47^{\prime \prime}$ & $4 \mathrm{~h} 48^{\prime} 26^{\prime \prime}$ & 1.4 \\
\hline
\end{tabular}

space point, then LHAPDF 6 is significantly faster since it does not need to interpolate an extra 12 values which will not be used. Legacy code written to use the PDFLIB or LHAPDF 5 interfaces is often structured to make use of this feature, and such code may be slightly slower with LHAPDF 6. However, where code can be rewritten to make use of a single-flavour approach, significant speed-ups can be achieved, as shown in Table 3 which gives timing information obtained with the Sherpa event generator [28]. Retrospective PDF reweighting operations using the LHAPDF6 API, as described in Sect. 7, should see particularly noticeable performance increases with LHAPDF 6, since the initial-state parton IDs are already known and hence only two parton flavours need to be evolved per event.

For code which has not been rewritten to use the LHAPDF 6 API, a performance improvement may be implemented in a future LHAPDF 6 version, explicitly adding caching of positional interpolation weights between evolution calls, so that consecutive evaluations at the same phase space point do not need to fully recompute the PDF interpolation. In an extreme case all required PDF derivatives at grid knots could also be pre-computed, similarly to how the knot point $\log x$ and $\log Q^{2}$ are currently computed during PDF initialisation; however, this would be likely to introduce a memory bottleneck in the computation, and methods such as use of space-filling curves to optimise CPU cache usage would add significant complication.
Additional CPU performance improvements are also being considered, in particular use of vectorised (and currently CPU-architecture-specific) SSE or AVX instructions for parallel interpolation of all flavours, or multiple simultaneous PDF queries. Vectorisation works best when there are no conditional branchings, hence re-engineering the spline interpolation to make best use of vectorisation would involve removing the current if-branching used to identify the edges of $Q$ subgrids and instead using extrapolated "halos" surrounding each subgrid. However, such an approach may have numerical consequences, particularly in how the edges of the grid and hand-over to extrapolation is handled, and will not be taken lightly. We welcome feedback on the extent to which particle physics computations are CPU-bound by LHAPDF interpolation.

Equivalent concerns apply to the possibility to use generalpurpose graphical processing units (GP GPUs) for vectorised PDF evolution; parallel evolution of $\mathcal{O}(13)$ parton flavours would not justify the trade-off of extra GPU code-complexity and platform-specificity. An alternative use would be to compute many points in parallel, but this is often not a natural use since many applications are Markov Chains where the next step is conditional on the result at the current one. It could benefit PDF reweighting, however, and should GPU implementations of matrix element event generation codes become prevalent $[37,38]$ then it will be natural for LHAPDF to support GPU operation. For the time being we prefer not to prematurely optimise for use-cases which may not manifest.

Parallel execution at the multi-thread level, or across multiple processes with shared read-only memory, may also be useful in PDF reweighting and does not have the technical overhead of GPGPU programming. LHAPDF 6 does not include any specific mechanisms to interface with multi-core frameworks such as OpenMP or MPI, but is largely safe to use with applications written to use them. Since there is some global state for the global configuration and the PDFSet objects created and returned by LHAPDF: : getPDFSet (), LHAPDF 6 is not $100 \%$ thread-safe; but if all changes to global and set-level configuration are made before the concurrent block, then use of PDF querying operations on PDF objects allocated locally to each thread should be safe.

A final, usually very minor, speed improvement has been seen in the initialisation time of LHAPDF6 PDF members. Since members are now located in individual files rather than within one large file for the whole set, random access to a particular PDF no longer requires "scrolling" through the rest of the file and loading the rest of the set's members. This speed improvement is not usually noticeable because the time taken to load a PDF set in either LHAPDF version is far less than $1 \mathrm{~s}$. Some unusual applications may need to reload PDFs from file very frequently, however, and for such situations we 
have made use of a custom fast parser of numeric data from ASCII files, where the speed-up is achieved by ignoring the possibility of wide-character types (e.g. Unicode) which are implicitly handled by C++'s I/O stream types. This optimisation makes LHAPDF 6 loading of whole PDF sets as fast as in LHAPDF 5. A further speed-up at initialisation time, if really desired, can be achieved by zipping the PDF set directories into . zip files - this trick is described in the next section, since the main effect is upon disk space rather than significant speed improvements.

\subsection{Disk space requirements}

The disk space requirements of LHAPDF 6 data sets are largely similar to those of their LHAPDF 5 equivalents. For example, the CT10nlo PDF set file is $21 \mathrm{MB}$ in LHAPDF 5 and the equivalent LHAPDF 6 directory contains $33 \mathrm{MB}$ of data files; showing the opposite trend, the NNPDF 2.3 NLO PDFs are all typically $95 \mathrm{MB}$ in LHAPDF 5 and $84 \mathrm{MB}$ in LHAPDF 6.

LHAPDF 6's use of directories and member-specific data files within does permit an extreme disk-space optimisation where dat files which will not be used can be removed from the set directory. This is not recommended in typical usage, but may be found to be helpful when e.g. the central members of many PDF sets need to be available, but error sets are not needed at all. A less extreme optimisation is to compress each . dat grid data file into .dat.zip or . dat.gz file and use the zlibc library to access them as if they were unzipped. This can be done without modifying any code by (on Linux systems) setting the \$LD_PRELOAD environment variable to the path to zlibc's uncompress.so library, and the typical compression factor of 3-4 reduces the disk space needed to store the data and can also speed up PDF initialisation. There are typically too many portability issues with this approach to currently make zipped data files standard in LHAPDF 6, but the option exists for applications which need it.

\section{PDF migration and validation}

A major task, as substantial as writing the new library, has been the migration of PDFs from the multitude of LHAPDF 5 formats to the new GridPDF format and interpolator, and then validating their faithfulness to the originals. This has been done in several steps, starting with a Python script which used the LHAPDF 5 interface (with some extensions) to extract the grid knots and dump the PDF data at the original knot points into the new format. This script has undergone extensive iteration, as support was added for subgrids, memberspecific metadata, etc., and to allow more automation of the conversion process for hundreds of PDFs.
The choice was made to only convert the most recent PDF sets in each family unless there were specific requests for earlier ones: this collection is more than 200 PDF sets, and only a few older PDFs have been requested in addition to the latest sets.

To validate PDFs, a comparison system was developed, using a $\mathrm{C}++$ code to dump PDF $x f$ values in scans across $\log x$ and $\log Q$ (as well as $\alpha_{\mathrm{S}}$ values in $\log Q$ ) in the ranges $x \in\left[10^{-10}, 1\right]$ and $Q \in\left[1,10^{4}\right] \mathrm{GeV}$, with 10 sample points per decade in each variable. For scans in $x$, fixed values of $Q \in\{10,50,100,200,500,1000,2000,5000\} \mathrm{GeV}$ were used, and for the scans in $Q$, fixed $x \in\left\{10^{-8}, 10^{-6}, 10^{-4}\right.$, $\left.10^{-2}, 0.1,0.2,0.5,0.8\right\}$ were used. The same $\mathrm{C}++$ code was used - with some compile-time specialisation - to dump values from both LHAPDF 5 and 6, to ensure exactly equivalent treatment of the two versions.

The corresponding data files from each version were then compared to each other using a difference metric which corresponds to the fractional deviation of the v6 value from the original v5 one in regions where the $x f$ value is large, but which suppresses differences as the PDFs go to zero, to minimise false alarms. An ad hoc difference tolerance of $10^{-3}$ was chosen on consultation with PDF authors as a level to which no-one would object, despite differences in opinion on e.g. preferred interpolation schemes. This level, as illustrated in Fig. 1 for the CT10nlo central PDF member validation, has been achieved almost everywhere for the majority of PDFs. Several differences were found this way, which helped with debugging the LHAPDF 6 code, the migration system, and occasionally the numerical stability of the original PDF's interpolation grid.

Before being officially made available for download from the LHAPDF website and AFS \& CVMFS locations, the validation plots resulting from this process had to be checked by the original set authors as well as the LHAPDF 6 team. To date more than 200 PDF sets, from the ATLAS, CTEQ \& CJ [39,40], HERAPDF [41], MRST [12,42,43], MSTW [4446], and NNPDF [47,48] fitting collaborations, have been approved in this way. In addition, over 100 new sets have been supplied directly to LHAPDF in the new native data format from the JR [49], METAPDF [50], MMHT [51], and NNPDF [52] collaborations. Tools to help with PDF migration from LHAPDF 5 and validation of migrated or independently constructed PDFs may be found in the migration subdirectory of the LHAPDF source package, but only in the developers' version available from the Mercurial repository.

\section{Summary and prospects}

After a lengthy public testing period, the first official LHAPDF 6 version, 6.0.0, was released in August 2013. As described, this new version of LHAPDF maintains compat- 

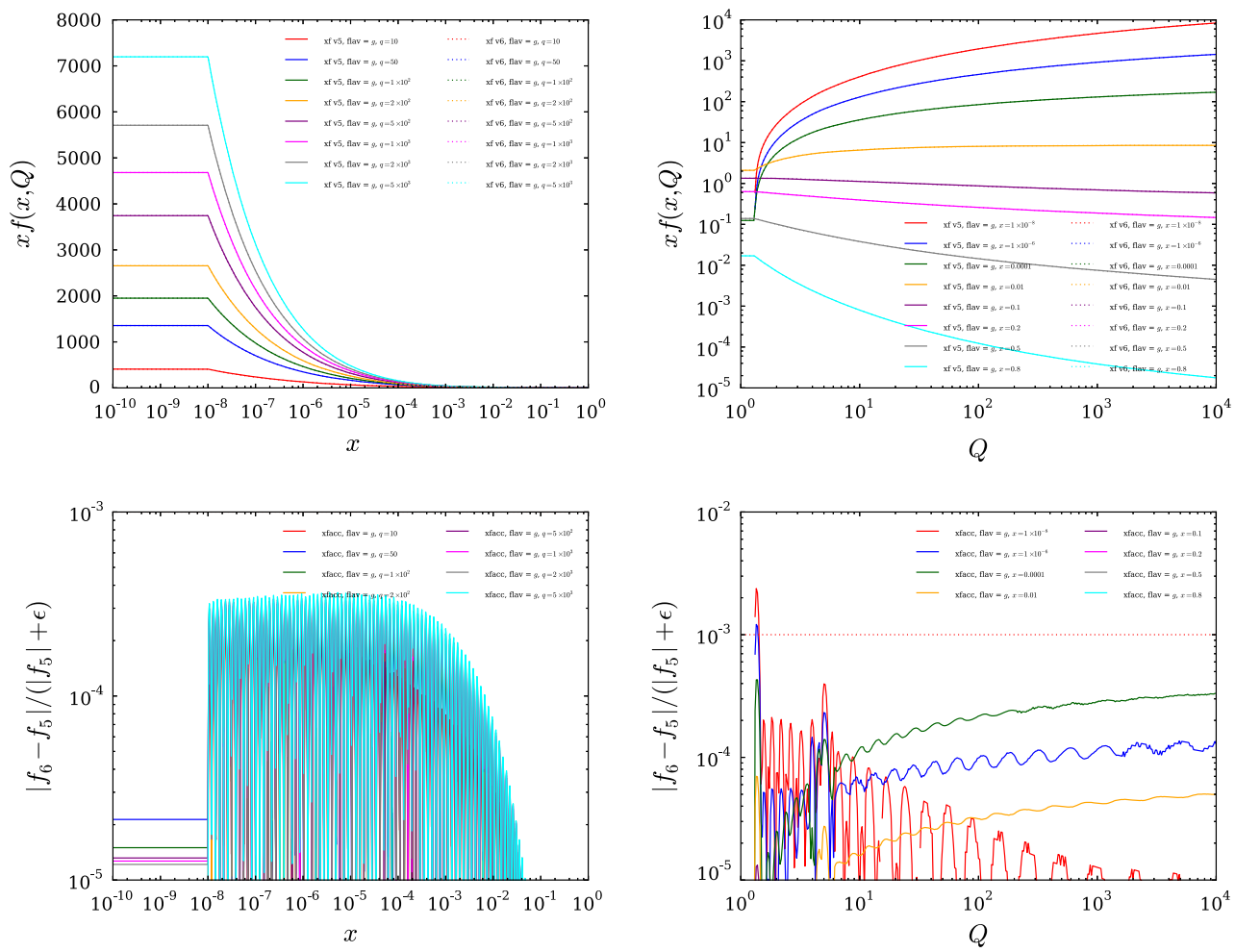

Fig. 1 Example comparison plots for the validation of the CT10nlo [39] central gluon PDF, showing the PDF behaviour as a function of $x$ on the left and $Q$ on the right. The upper plots show the actual PDF shapes with both the v5 and v6 versions overlaid, and the lower plots contain plots of the corresponding v5 vs. v6 regularised accuracy metrics. The differences between $\mathrm{v} 5$ and $\mathrm{v} 6$ cannot be seen in the upper

ibility with applications written to use the LHAPDF 5 code interfaces, while providing much more powerful models for dynamic allocation of PDF memory and for parton density metadata.

The new design also provides a unified data format and routines for PDF interpolation, which decouples new releases of PDF sets from the slower release cycle of the LHAPDF software library. The new design which allows very general parton content has also proven useful for the new generation of NNPDF sets which include polarised partons and photon constituents [53,54], and for implementing fragmentation functions using the PDF interpolation machinery. Several PDF sets have already been supplied directly to the LHAPDF 6 library in the new native format, which simplifies and speeds up the release of new PDFs for PDF users and authors alike.

The new code design vastly reduces the memory requirements of the library compared to the several GB demanded by LHAPDF 5, meaning that it can efficiently use multiple full PDF sets at the same time - a task which was unfeasible with Grid-distributed builds of LHAPDF 5. Gains in CPU performance, although a smaller effect than the fix to LHAPDF 5's

plots, since the fractional differences are everywhere below one part in 1000 except right at the very lowest $Q$ point where the two PDFs freeze in very slightly different ways. The oscillatory difference structures arise from small differences in the interpolation between the identical interpolation knots

pathological memory requirements, are also possible with the new structure due to the ability to interpolate single flavours at a time rather than being forced to always evolve all of a PDF's constituent flavours at the same time: this particularly improves performance in reweighting applications where at most two parton flavours need to be evolved per event. There is room for further CPU performance improvements by adding explicit caching of some interpolation coefficients at a given $\left(x, Q^{2}\right)$ point, and with more work the code can be optimised to allow use of vectorised CPU instructions. Addition of flavour aliasing or compressed data file reading could reduce the data size on disk. However, all such performance optimisations need to be judged according to the real-world benefits which they offer, against the code complexity which they typically introduce.

Finally, LHAPDF 6 provides new tools for PDF uncertainty and reweighting calculations, to respond to the increasingly complex ways in which particle physics experiment and phenomenology use PDFs.

At present the scope of LHAPDF 6 is intentionally more LHC-focused than LHAPDF 5. Accordingly, no QCD evolution is planned for the library since this functionality ended 
up virtually unused in LHAPDF 5. Several quality external libraries $[8,55,56]$ exist to perform this evolution and generate the grid files - or if desired, the PDF class can be derived from to call an evolution library at runtime. Similarly, there is at present no plan to support resolved virtual photon structure functions or transverse-momentum-dependent PDFs, which require additional parameters in the interpolation space.

Nuclear corrections to nucleon PDFs are also not currently supported in a transparent way, but this is planned for a nearfuture LHAPDF version. In the meantime, external nuclear correction factors such as the EPS sets [57] can be applied explicitly to nucleon PDFs from LHAPDF. Nuclear PDFs with the corrections already "hard-coded" into LHAPDF 6 grids are also trivially supported, since these are indistinguishable from nucleon PDFs, other than via the Particle metadata key which can declare the nucleus/ion as the parent particle in place of the usual proton - this is another strength of the decision to use the standard PDG particle ID number scheme in LHAPDF 6.

In summary, LHAPDF 6 is fully operational at the planned level, offers very significant improvements in performance and capabilities over LHAPDF 5, and is recommended as the production version of LHAPDF in the high-precision era of collider physics which begins with LHC Run 2.

Acknowledgments Thanks to Jeppe Andersen, Juan Rojo, Luigi del Debbio, Richard Ball, and Nathan Hartland for helpful suggestions and inputs on PDF collaboration requirements, which were invaluable in evolving this design. Many thanks also to David Hall, who provided the Ihapdf data management script, to David Mallows for early help with the interpolator code and Python interface, and to Gavin Salam for several suggestions and a fast numeric ASCII parser code. AB wishes to acknowledge support from a Royal Society University Research Fellowship, a CERN Scientific Associateship, and IPPP Associateships during the period of LHAPDF6 development. IPPP grants also supported the work of SL, MR, and David Mallows on this project. KN thanks the University of Glasgow College of Science \& Engineering for a $\mathrm{PhD}$ studentship scholarship.

Open Access This article is distributed under the terms of the Creative Commons Attribution License which permits any use, distribution, and reproduction in any medium, provided the original author(s) and the source are credited.

Funded by $\mathrm{SCOAP}^{3}$ / License Version CC BY 4.0.

\section{References}

1. H. Plothow-Besch, PDFLIB: a Library of all available parton density functions of the nucleon, the pion and the photon and the corresponding alpha-s calculations. Comput. Phys. Commun. 75, 396416 (1993)

2. O.S. Bruning, P. Collier, P. Lebrun, S. Myers, R. Ostojic et al., LHC Design Report. 1. The LHC Main Ring, CERN-2004-003-V1, CERN-2004-003
3. J.M. Campbell, J. Huston, W. Stirling, Hard interactions of quarks and gluons: a primer for LHC physics. Rep. Prog. Phys. 70, 89 (2007). arXiv:hep-ph/0611148

4. S. Forte, G. Watt, Progress in the determination of the partonic structure of the proton. Ann. Rev. Nucl. Part. Sci. 63, 291-328 (2013). arXiv:1301.6754

5. M. Whalley, D. Bourilkov, R. Group, The Les houches accord PDFs (LHAPDF) and LHAGLUE. arXiv:hep-ph/0508110

6. D. Bourilkov, R.C. Group, M.R. Whalley, LHAPDF: PDF use from the tevatron to the LHC. arXiv:hep-ph/0605240

7. W. Giele, E.N. Glover, I. Hinchliffe, J. Huston, E. Laenen et al., The QCD/SM working group: summary report. arXiv:hep-ph/0204316

8. M. Botje, QCDNUM: fast QCD evolution and convolution. Comput. Phys. Commun. 182, 490-532 (2011). arXiv:1005.1481

9. S. Frixione, B.R. Webber, Matching NLO QCD computations and parton shower simulations. JHEP 0206, 029 (2002). arXiv:hep-ph/0204244

10. S. Frixione, P. Nason, C. Oleari, Matching NLO QCD computations with parton shower simulations: the POWHEG method. JHEP 0711, 070 (2007). arXiv:0709.2092

11. R. Frederix, S. Frixione, V. Hirschi, F. Maltoni, R. Pittau et al., Fourlepton production at hadron colliders: aMC@ NLO predictions with theoretical uncertainties. JHEP 1202, 099 (2012). arXiv: 1110.4738

12. A. Martin, R. Roberts, W. Stirling, R. Thorne, Parton distributions incorporating QED contributions. Eur. Phys. J. C 39, 155-161 (2005). arXiv:hep-ph/0411040

13. E.L. Berger, P.M. Nadolsky, F.I. Olness, J. Pumplin, Light gluino constituents of hadrons and a global analysis of hadron scattering data. Phys. Rev. D 71, 014007 (2005). arXiv:hep-ph/0406143

14. Particle Data Group Collaboration, J. Beringer et al., Review of particle physics (RPP). Phys. Rev. D 86, 010001 (2012)

15. T. van Ritbergen, J.A.M. Vermaseren, S.A. Larin, The four loop beta function in quantum chromodynamics. Phys. Lett. B 400, 379384 (1997). arXiv:hep-ph/9701390

16. K.G. Chetyrkin, J.H. Kuhn, C. Sturm, QCD decoupling at four loops. Nucl. Phys. B 744, 121-135 (2006). arXiv:hep-ph/0512060

17. K.G. Chetyrkin, B.A. Kniehl, M. Steinhauser, Strong coupling constant with flavor thresholds at four loops in the modified minimalsubtraction scheme. Phys. Rev. Lett. 79, 2184-2187 (1997)

18. B. Schmidt, M. Steinhauser, CRunDec: a C++ package for running and decoupling of the strong coupling and quark masses. Comput. Phys. Commun. 183, 1845-1848 (2012). arXiv:1201.6149

19. YAML 1.2: YAML Ain't Markup Language. http://yaml.org

20. yaml-cpp: a YAML parser and emitter for C++. https://code. google.com/p/yaml-cpp/

21. LHAPDF website. https://lhapdf.hepforge.org

22. G. Watt, Parton distribution function dependence of benchmark Standard Model total cross sections at the 7 TeV LHC. JHEP 1109, 069 (2011). arXiv: 1106.5788

23. G. Watt, R.S. Thorne, Study of Monte Carlo approach to experimental uncertainty propagation with MSTW 2008 PDFs. JHEP 1208, 052 (2012). arXiv: 1205.4024

24. NNPDF Collaboration, R.D. Ball et al., Reweighting NNPDFs: the W lepton asymmetry. Nucl. Phys. B 849, 112-143 (2011). arXiv: 1012.0836

25. R.D. Ball, V. Bertone, F. Cerutti, L. Del Debbio, S. Forte et al., Reweighting and unweighting of parton distributions and the LHC W lepton asymmetry data. Nucl. Phys. B 855, 608-638 (2012). arXiv: 1108.1758

26. H. Paukkunen, P. Zurita, PDF reweighting in the Hessian matrix approach. JHEP 1412, 100 (2014). arXiv: 1402.6623

27. M. Botje, J. Butterworth, A. Cooper-Sarkar, A. de Roeck, J. Feltesse et al., The PDF4LHC Working Group Interim Recommendations. arXiv:1101.0538 
28. T. Gleisberg, S. Hoeche, F. Krauss, M. Schonherr, S. Schumann et al., Event generation with SHERPA 1.1. JHEP 0902, 007 (2009). arXiv:0811.4622

29. S. Alioli, P. Nason, C. Oleari, E. Re, A general framework for implementing NLO calculations in shower Monte Carlo programs: the POWHEG BOX. JHEP 1006, 043 (2010). arXiv:1002.2581

30. J. Alwall, R. Frederix, S. Frixione, V. Hirschi, F. Maltoni et al., The automated computation of tree-level and next-to-leading order differential cross sections, and their matching to parton shower simulations. JHEP 1407, 079 (2014). arXiv: 1405.0301

31. J. Pumplin, D. Stump, J. Huston, H. Lai, P.M. Nadolsky et al., New generation of parton distributions with uncertainties from global QCD analysis. JHEP 0207, 012 (2002). arXiv:hep-ph/0201195

32. T. Sjostrand, S. Mrenna, P.Z. Skands, PYTHIA 6.4 physics and manual. JHEP 0605, 026 (2006). arXiv:hep-ph/0603175

33. CTEQ Collaboration, H. Lai et al., Global QCD analysis of parton structure of the nucleon: CTEQ5 parton distributions. Eur. Phys. J. C 12, 375-392 (2000). arXiv:hep-ph/9903282

34. T. Sjostrand, S. Mrenna, P.Z. Skands, A brief introduction to PYTHIA 8.1. Comput. Phys. Commun. 178, 852-867 (2008). arXiv:0710.3820

35. G. Corcella, I. Knowles, G. Marchesini, S. Moretti, K. Odagiri et al., HERWIG 6: an event generator for hadron emission reactions with interfering gluons (including supersymmetric processes). JHEP 0101, 010 (2001). arXiv:hep-ph/0011363

36. M. Bahr, S. Gieseke, M. Gigg, D. Grellscheid, K. Hamilton et al., Herwig++ physics and manual. Eur. Phys. J. C 58, 639-707 (2008). arXiv:0803.0883

37. K. Hagiwara, J. Kanzaki, N. Okamura, D. Rainwater, T. Stelzer, Fast calculation of HELAS amplitudes using graphics processing unit (GPU). Eur. Phys. J. C 66, 477-492 (2010). arXiv:0908.4403

38. W. Giele, G. Stavenga, J.-C. Winter, Thread-scalable evaluation of multi-jet observables. Eur. Phys. J. C 71, 1703 (2011). arXiv: 1002.3446

39. H.-L. Lai, M. Guzzi, J. Huston, Z. Li, P.M. Nadolsky et al., New parton distributions for collider physics. Phys. Rev. D 82, 074024 (2010). arXiv:1007.2241

40. A. Accardi, J. Owens, W. Melnitchouk, The CJ12 parton distributions, PoS DIS2013, 040 (2013)

41. H1 Collaboration, ZEUS Collaboration, V. Radescu, Hera precision measurements and impact for LHC predictions. arXiv:1107.4193
42. A. Sherstnev, R. Thorne, Parton distributions for LO generators. Eur. Phys. J. C 55, 553-575 (2008). arXiv:0711.2473

43. A. Sherstnev, R. Thorne, Different PDF approximations useful for LO Monte Carlo generators. arXiv:0807.2132

44. A. Martin, W. Stirling, R. Thorne, G. Watt, Parton distributions for the LHC. Eur. Phys. J. C 63, 189-285 (2009). arXiv:0901.0002

45. A. Martin, W. Stirling, R. Thorne, G. Watt, Uncertainties on $\alpha_{S}$ in global PDF analyses and implications for predicted hadronic cross sections. Eur. Phys. J. C 64, 653-680 (2009). arXiv:0905.3531

46. A. Martin, W. Stirling, R. Thorne, G. Watt, Heavy-quark mass dependence in global PDF analyses and 3- and 4-flavour parton distributions. Eur. Phys. J. C 70, 51-72 (2010). arXiv:1007.2624

47. NNPDF Collaboration, R.D. Ball et al., Unbiased global determination of parton distributions and their uncertainties at NNLO and at LO. Nucl. Phys. B 855, 153-221 (2012). arXiv:1107.2652

48. R.D. Ball, V. Bertone, S. Carrazza, C.S. Deans, L. Del Debbio et al., Parton distributions with LHC data. Nucl. Phys. B 867, 244-289 (2013). arXiv:1207.1303

49. P. Jimenez-Delgado, Delineating the polarized and unpolarized partonic structure of the nucleon. arXiv: 1410.2431

50. J. Gao, P. Nadolsky, A meta-analysis of parton distribution functions. JHEP 1407, 035 (2014). arXiv:1401.0013

51. L. Harland-Lang, A. Martin, P. Motylinski, R. Thorne, Parton distributions in the LHC era: MMHT 2014 PDFs. arXiv:1412.3989

52. The NNPDF Collaboration, R.D. Ball et al., Parton distributions for the LHC Run II. arXiv:1410.8849

53. NNPDF Collaboration, E.R. Nocera, R.D. Ball, S. Forte, G. Ridolfi, J. Rojo, A first unbiased global determination of polarized PDFs and their uncertainties. Nucl. Phys. B 887, 276-308 (2014). arXiv: 1406.5539

54. NNPDF Collaboration, R.D. Ball et al., Parton distributions with QED corrections. Nucl. Phys. B 877, 290-320 (2013). arXiv: 1308.0598

55. G. Salam, J. Rojo, The HOPPET NNLO parton evolution package. arXiv:0807.0198

56. V. Bertone, S. Carrazza, J. Rojo, APFEL: a PDF evolution library with QED corrections. Comput. Phys. Commun. 185, 1647-1668 (2014). arXiv: 1310.1394

57. K. Eskola, H. Paukkunen, C. Salgado, EPS09: a new generation of NLO and LO nuclear parton distribution functions. JHEP 0904, 065 (2009). arXiv:0902.4154 